\title{
Evaluation of state integrals at rational points
}

\author{
Stavros Garoufalidis and Rinat Kashaev
}

\begin{abstract}
Multi-dimensional state-integrals of products of Faddeev's quantum dilogarithms arise frequently in Quantum Topology, quantum Teichmüller theory and complex Chern-Simons theory. Using the quasi-periodicity property of the quantum dilogarithm, we evaluate 1-dimensional state-integrals at rational points and express the answer in terms of the Rogers dilogarithm, the cyclic (quantum) dilogarithm and finite state-sums at roots of unity. We illustrate our results with the evaluation of the state-integrals of the $4_{1}, 5_{2}$ and $(-2,3,7)$ pretzel knots at rational points.
\end{abstract}

1 Introduction

1.1 State-integrals and their $q$-series

1.2 The Rogers and the cyclic dilogarithms

1.3 Evaluation of state-integrals

1.4 The quantum dilogarithm at roots of unity

Acknowledgments

2 Integrals of quasi-periodic functions

2.2 Applications to 1-dimensional state-integrals: proof of Theorem 1.1

Key words and phrases: state-integrals, q-series, quantum dilogarithm, cyclic dilogarithm, Rogers dilogarithm, quasi-periodic functions, Nahm equation, gluing equations, $4_{1}, 5_{2},(-2,3,7)$ pretzel knot. 
2.3 The case of the $(-2,3,7)$ pretzel knot

3 Proof of Theorem $\mathbf{1 . 9}$

Appendix A Some useful properties of the quantum dilogarithm

Appendix B The Teichmüller TQFT partition function of the $(-2,3,7)$ pretzel knot

B.1 Calculation of $K(X, y)$

B.2 Proof of Theorem B.1

\section{Introduction}

\subsection{State-integrals and their $q$-series}

State-integrals are multi-dimensional integrals of products of Faddeev's quantum dilogarithms. They appear in abundance in Quantum Topology, quantum Teichmüller theory and in complex Chern-Simons theory. State integrals were studied among others by Hikami [Hik01], Dimofte-GukovLennels-Zagier [DGLZ09], Andersen-Kashaev [AK14, AK], Kashaev-LuoVartanov [KLV12], Dimofte [Dim14a] and Dimofte-Garoufalidis [Dim14b].

In our previous paper $[\mathrm{GK}]$, we showed how to express 1-dimensional state-integrals as a finite sum of products of $q$-series and $\tilde{q}$-series with integer coefficients, where the variables $q$ and $\tilde{q}$ are related by the modular transformation: $q=e^{2 \pi \mathrm{i} \tau}$ and $\tilde{q}=e^{-2 \pi \mathrm{i} / \tau}$.

In this paper we evaluate 1-dimensional state-integrals at rational points in terms of the Rogers dilogarithm, the cyclic (quantum) dilogarithm of [FK94] and truncated state-sums at roots of unity. Our formulas are syntactically similar with

(a) the constant terms of the power series that appear in the Quantum Modularity Conjecture of Zagier [Zag10, GZa],

(b) the 1-loop terms of the perturbation expansion of complex ChernSimons theory [Dim14b], 
(c) the state-sums of quantum Teichmüller theory [Kas94, Kas95, Kas97] and also [BB07, Sec.6].

This is not a coincidence; it is one part of a story discussed in detail in [GZb].

In order to keep our principle clear, we focus exclusively on 1-dimensional state-integrals, and we illustrate our results for the state-integrals of $4_{1}, 5_{2}$ and $(-2,3,7)$ pretzel knots. In a separate publication we will discuss the evaluation of multi-dimensional state-integrals.

A 1-dimensional state-integral is an absolutely convergent integral of the form

$$
\mathcal{I}_{A, B}(\mathrm{~b})=\int_{\mathbb{R}+\mathrm{i} \epsilon} \Phi_{\mathrm{b}}(x)^{B} e^{-A \pi \mathrm{i} x^{2}} d x
$$

for a complex number $\mathrm{b}$ with $\mathrm{b}^{2} \notin \mathbb{R}_{\leq 0}$. Here $A, B$ are natural numbers satisfying $B>A>0$ and $\Phi_{\mathrm{b}}(x)$ is Faddeev's quantum dilogarithm function [Fad95]. Few properties of this special function are reviewed in Appen$\operatorname{dix} \mathrm{A}$.

A numerical computation by the first author and Zagier [GZb] suggested the following formula for $\mathcal{I}_{1,2}(1)$ :

$$
\mathcal{I}_{1,2}(1)=\frac{e^{\pi \mathrm{i} / 6}}{\sqrt{3}}\left(e^{\frac{V}{2 \pi}}-e^{-\frac{V}{2 \pi}}\right)
$$

(and more generally for the Taylor coefficients of the analytic function $\mathcal{I}_{1,2}$ (b) at $\mathrm{b}=1)$, where $V=2 \operatorname{Im}\left(\operatorname{Li}_{2}\left(e^{\pi \mathrm{i} / 3}\right)\right)=2.0298832 \cdots$ is the volume of the $4_{1}$ knot. Understanding and proving the above identity led to the results of our paper.

Our aim is to evaluate $\mathcal{I}_{A, B}(\mathrm{~b})$ when $\mathrm{b}^{2}=M / N$ for a pair of coprime natural numbers $M, N$. The content of our paper can be summarized in a diagram

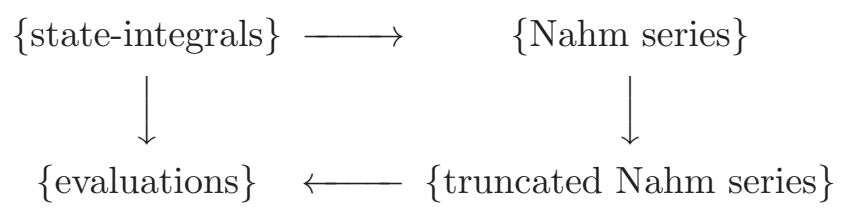

The top arrow was the content of our previous article [GK]. To recall the connection between state-integrals and $q$-series, consider the integrand of the state-integral $\mathcal{I}_{A, B}(\mathrm{~b})$, shifted by $c_{\mathrm{b}}=\mathrm{i}\left(\mathrm{b}+\mathrm{b}^{-1}\right) / 2$ :

$$
\mathrm{f}\left(x-c_{\mathrm{b}}\right)=\Phi_{\mathrm{b}}(x)^{B} e^{-A \pi \mathrm{i} x^{2}} .
$$


The quasi-periodicity of the quantum dilogarithm (see Equations (A.4a)(A.4b)) implies that

$$
\mathrm{f}\left(x+\mathrm{i} m \mathrm{~b}+\mathrm{i} n \mathrm{~b}^{-1}\right)=\mathrm{f}(x) \mathrm{g}_{m}^{+}\left(e^{2 \pi \mathrm{b} x}, q_{+}\right) \mathrm{g}_{n}^{-}\left(e^{2 \pi \mathrm{b}^{-1} x}, q_{-}\right)
$$

where $q_{ \pm}=e^{2 \pi \mathrm{ib}^{ \pm 2}}$ and

$$
\mathrm{g}_{k}^{ \pm}(x, q)=(-x)^{A k} \frac{q^{\frac{A}{2} k(k+1)}}{(q x ; q)_{k}^{B}} .
$$

This gives rise to the series $G^{ \pm}(x, q) \in \mathbb{Z} \llbracket x, q \rrbracket$ defined by

$$
G^{ \pm}(x, q)=\sum_{k=0}^{\infty} \mathrm{g}_{k}^{ \pm}(x, q)
$$

The $q$-series $G^{ \pm}(1, q) \in \mathbb{Z} \llbracket q \rrbracket$ are special $q$-hypergeometric series of Nahm type and appear in the expression of the state-integral $\mathcal{I}_{A, B}(b)$ as a sum of products of $q$-series and $\tilde{q}$-series, where $\tilde{q}=1 / q_{-}$, see [GK, Thm.1.1].

Throughout the paper, $(M, N)$ will denote an admissible pair, i.e., a pair of coprime positive integers. Consider the state-sum defined by

$$
G_{M, N}\left(x_{+}, x_{-}\right)=\sum_{k=0}^{M N-1} \mathrm{~g}_{k P}^{+}\left(x_{+}, \zeta_{N}^{M}\right) \mathrm{g}_{k Q}^{-}\left(x_{-}, \zeta_{M}^{N}\right)
$$

where $P, Q$ are integers that satisfy the equation $M P+N Q=1$ and $\zeta_{N}=$ $e^{2 \pi i / N}$. When $x_{+}^{N}=x_{-}^{M}$, it follows from Lemma 2.2 that $G_{M, N}\left(x_{+}, x_{-}\right)$is independent of the choice of $P$ and $Q$. Observe that

$$
G_{1, N}\left(x_{+}, x_{-}\right)=G_{N}^{+}\left(x_{+}\right), \quad G_{M, 1}\left(x_{+}, x_{-}\right)=G_{M}^{-}\left(x_{-}\right)
$$

where

$$
G_{N}^{ \pm}(x)=\sum_{k=0}^{N-1} \mathrm{~g}_{k}^{ \pm}\left(x, \zeta_{N}\right)
$$

\subsection{The Rogers and the cyclic dilogarithms}

Recall the Rogers dilogarithm [Neu04, GZ07]

$$
\mathrm{R}(z)=\mathrm{Li}_{2}(z)+\frac{1}{2} \log (z) \log (1-z)-\frac{\pi^{2}}{6}
$$


and its extension as a multivalued function on the universal abelian cover of $\mathbb{C} \backslash\{0,1\}$.

The cyclic (quantum) dilogarithm $\mathrm{D}_{N}(x ; q)$ is the $N$-th root of a polynomial in $x$ with constant term 1 defined by

$$
\mathrm{D}_{N}(x ; q)=\prod_{k=1}^{N-1}\left(1-q^{k} x\right)^{k / N}
$$

It appeared in [KMS93, Eqn.C.3] and [Kas99, Eqn.2.30], and its $N$-th power is characterized among polynomials by the functional equation

$$
\frac{\mathrm{D}_{N}\left(\zeta_{N} x ; \zeta_{N}\right)^{N}}{\mathrm{D}_{N}\left(x ; \zeta_{N}\right)^{N}}=\frac{(1-x)^{N}}{1-x^{N}}, \quad \mathrm{D}_{N}(0)^{N}=1 .
$$

It will be useful to introduce the following variant $\not_{N}$ defined by

$$
\not D_{N}(x ; q)=\prod_{k=1}^{N}\left(1-x q^{k}\right)^{k / N}=\left(1-x q^{N}\right) \mathrm{D}_{N}(x ; q) \text {. }
$$

\subsection{Evaluation of state-integrals}

Our main theorem evaluates the state-integral at $\mathrm{b}^{2}=M / N$ in terms of the state-sums $G_{M, N}$, the Rogers dilogarithm and the cyclic dilogarithm.

Fix an admissible pair $(M, N)$, and define

$$
\mathrm{b}=\sqrt{M / N}, \quad \mathrm{~s}=\sqrt{M N} .
$$

Let

$$
\mathcal{S}=\left\{w \mid \mathrm{g}\left(e^{2 \pi \mathrm{s} w}\right)=1,0<\mathrm{s} \operatorname{Im}(w)-\lambda<1\right\}
$$

where $\lambda$ is a generic real number such that

$$
-(M+N) / 2<\lambda<0
$$

and

$$
\mathrm{g}(z)=(-z)^{A}(1-z)^{-B} \in \mathbb{Q}\left[z^{ \pm 1}\right]
$$

Note that if $w \in \mathcal{S}$, then $e^{2 \pi s w}$ is an algebraic number with a fixed choice of $N$ and $M$-th roots. 
Theorem 1.1. When $\mathrm{b}^{2}=M / N$ we have:

$$
\begin{aligned}
\mathcal{I}_{A, B}(\mathrm{~b})= & e^{\pi \mathrm{i} \frac{B+3 A(M+N+1)^{2}-6 M N}{12 M N}} \mathrm{~s}^{-1} \\
& \cdot \sum_{w \in \mathcal{S}} \frac{e^{\frac{i B}{2 \pi \mathrm{s}^{2}} \mathrm{R}(z)}(1-z)^{\frac{(2 N+1)(2 M+1)}{4 M N}} B}{z \mathrm{~g}^{\prime}(z) \not D_{N}\left(\theta_{+}, q_{+}\right)^{B} \not D_{M}\left(\theta_{-}, q_{-}\right)^{B}} G_{M, N}\left(\theta_{+}, \theta_{-}\right),
\end{aligned}
$$

where

$$
\begin{aligned}
& z=e^{2 \pi \mathrm{s} w}, \quad \theta_{+}=e^{2 \pi \mathrm{b} w}=z^{1 / N}, \quad \theta_{-}=e^{2 \pi \mathrm{b}^{-1} w}=z^{1 / M}, \\
& q_{+}=\zeta_{N}^{M}, \quad q_{-}=\zeta_{M}^{N} .
\end{aligned}
$$

Note that when $g(z)=1$, we have

$$
\mathrm{g}^{\prime}(z)=A z^{-1}+B(1-z)^{-1} .
$$

Corollary 1.2. For $M=1$ we obtain that

$$
\begin{aligned}
\mathcal{I}_{A, B}(\mathbf{b})= & e^{\pi \mathrm{i} \frac{B+3 A(N+2)^{2}-6 N}{12 N}} \frac{1}{\sqrt{N}} \\
& \cdot \sum_{w \in \mathcal{S}} e^{\frac{i B}{2 \pi N} \mathrm{R}(z)} \frac{(1-z)^{\frac{2 N+3}{4 N} B}}{(A+B z /(1-z)) D_{N}\left(\theta_{+}, q_{+}\right)^{B}} G_{N}^{+}\left(\theta_{+}\right) .
\end{aligned}
$$

When $M=N=1$ we obtain that

$$
\mathcal{I}_{A, B}(1)=e^{\pi \mathrm{i} \frac{B+3 A-6}{12}} \sum_{w \in \mathcal{S}} e^{\frac{i B}{2 \pi} \mathrm{R}(z)} \frac{(1-z)^{\frac{B}{4}}}{(A+B z /(1-z))} .
$$

Let us denote

$$
\mathrm{e}(x)=e^{2 \pi \mathrm{i} x}
$$


Corollary 1.3. When $M=N=1$ and $(A, B)=(1,2)$, we choose $\lambda$ to be a negative real number near zero,

$$
\begin{aligned}
\mathrm{g}(z) & =-z(1-z)^{-2} \\
\mathcal{S} & =\{\mathrm{i} / 6,5 \mathrm{i} / 6\} \\
z_{ \pm} & =\mathrm{e}( \pm 1 / 6) \\
e^{\pi \mathrm{i} \frac{B+3 A-6}{12}} & =\mathrm{e}\left(-\frac{1}{24}\right) \\
\left(e^{\frac{\mathrm{i} B}{2 \pi} \mathrm{R}\left(z_{+}\right)}, e^{\frac{\mathrm{iB}}{2 \pi} \mathrm{R}\left(z_{-}\right)}\right) & =\left(e^{-C} \mathrm{e}\left(-\frac{1}{24}\right),-e^{C} \mathrm{e}\left(-\frac{1}{24}\right) \mathrm{e}\left(\frac{1}{3}\right)\right) \\
\frac{\left(1-z_{ \pm}\right)^{\frac{B}{4}}}{\left(A+B z_{ \pm} /\left(1-z_{ \pm}\right)\right)} & =\frac{1}{\sqrt{3}} \mathrm{e}\left(\mp \frac{1}{3}\right)
\end{aligned}
$$

where $C=V /(2 \pi)$ and $V$ is the volume of the $4_{1}$ knot. When computing the Rogers dilogarithm of $z_{ \pm}$, keep in mind that we use the branches of the logarithm $\log z_{+}=2 \pi \mathrm{i} / 6$ and $\log z_{-}=10 \pi \mathrm{i} / 6$ dictated by Equations (12) and (13).

The above computation, combined with Equation (19) implies Equation (2). As was already mentioned, the proof of this equation was a main motivation for the results of our paper.

We now make few remarks about the number-theoretic, analytic and geometric properties of Equation (15).

Remark 1.4. It [GZa] (see also [Dim14b]) it was observed that although $\left(G^{+}\left(\theta_{+}\right)\right)^{N}$ and $\left(\not D_{N}\left(\theta_{+}, \zeta_{N}\right)\right)^{N}$ lie in the field $F_{G, N}=\mathbb{Q}\left(\theta_{+}, \zeta_{N}\right)$, their ratio lies in the smaller field $\mathbb{Q}\left(z, \zeta_{N}\right)$ (where $z$ satisfies $\mathrm{g}(z)=1$ ) which is an extension of $\mathbb{Q}(z)$ by $\zeta_{N}$. In particular, the above mentioned ratio is independent of the choice of the $N$-th root of $z$.

Remark 1.5. Although $\not_{N}$ is a multivalued function, the sum in Equation (15) is well-defined. This is a consequence of Theorem 1.9 below and the fact that the quantum dilogarithm is a meromorphic function.

Remark 1.6. When the state-integral is associated with a cusped hyperbolic manifold $M$, the set $\mathcal{S}$ is often in bijection with the set of nonabelian parabolic $\operatorname{PSL}(2, \mathbb{C})$ representations of $M$. Under such a bijection, the Rogers dilogarithm matches with the complex volume, and the value of $g^{\prime}(z)$ matches with the value of the 1-loop invariant of [DG13], suitably normalized. For an illustration, see Section 2.3. 
Remark 1.7. When the state-integral is associated with a cusped hyperbolic manifold $M$ and the identification of Remark 1.6 is available, one can identify Equation (15) with a sum of invariants of $M$ parametrized by nonabelian parabolic $\operatorname{PSL}(2, \mathbb{C})$ representations of $M$. Such invariants appear in Quantum Hyperbolic Geometry — see [Kas97] and also [BB07]. The invariants of Quantum Hyperbolic Geometry are defined up to multiplication by an $N$-root of unity. However, Equation (15) gives a well-defined relative choice of the $N$-th roots of unity. This is a consequence of the meromorphicity of the quantum dilogarithm.

Remark 1.8. As we already mentioned above, a numerical computation by the first author and Zagier suggests an explicit formula for the Taylor series of $\mathcal{I}_{1,2}$ (b) at $\mathrm{b}=1$ in terms of the asymptotics of the Kashaev invariant at $q=1$. We expect that the Taylor series of state-integrals at $\mathrm{b}=\sqrt{M / N}$ can be expressed in terms of the loop invariants of GaroufalidisDimofte [Dim14b]. We plan to study this in a later publication.

Theorem 1.1 follows from a lemma from complex analysis regarding integrals of quasi-periodic functions 2.3. This lemma is used twice, once to evaluate the quantum dilogarithm in terms of the cyclic dilogarithm, and another time to evaluate the state-integral $\mathcal{I}_{A, B}(\mathrm{~b})$.

\subsection{The quantum dilogarithm at roots of unity}

We fix an admissible pair $(M, N)$. Recall b and $\mathrm{s}$ from Equation (11). Let $\operatorname{Li}_{2}(z)=\sum_{n=1}^{\infty} \frac{z^{n}}{n^{2}}$ denote the Euler dilogarithm, defined for $|z|<1$ and analytically continued as a multivalued function on $\mathbb{C} \backslash\{0,1\}$.

Theorem 1.9. We have:

$$
\Phi_{\mathrm{b}}\left(\frac{z}{2 \pi \mathrm{s}}-c_{\mathrm{b}}\right)=\frac{e^{\frac{\mathrm{i}}{2 \pi \mathrm{s}^{2}} \operatorname{Li}_{2}\left(e^{z}\right)}\left(1-e^{z}\right)^{1+\frac{\mathrm{i} z}{2 \pi \mathrm{s}^{2}}}}{\mathrm{D}_{N}\left(e^{z / N} ; q_{+}\right) \mathrm{D}_{M}\left(e^{z / M} ; q_{-}\right)} .
$$

It is remarkable that the left-hand side is a meromorphic function of $z$ whereas the right hand side is assembled out of multivalued functions of $z$.

In particular when $M=1$, we obtain that

$$
\Phi_{\mathrm{b}}\left(x-c_{\mathrm{b}}\right)=\frac{e^{-\frac{1}{2 \pi i N} \operatorname{Li}_{2}\left(z^{N}\right)}}{\mathrm{D}_{N}(z)}\left(1-z^{N}\right)^{1+\frac{i x}{\sqrt{N}}}, \quad z=e^{2 \pi \mathrm{b} x},
$$


and when $M=N=1$, we obtain that

$$
\Phi_{1}(x)=\exp \left(\frac{\mathrm{i}}{2 \pi}\left(\operatorname{Li}_{2}\left(e^{2 \pi x}\right)+2 \pi x \log \left(1-e^{2 \pi x}\right)\right)\right)
$$

By using the equality

$$
\frac{\Phi_{\mathrm{b}}\left(\frac{z}{2 \pi \mathrm{s}}-c_{\mathrm{b}}\right)}{\Phi_{\mathrm{b}}\left(\frac{z}{2 \pi \mathrm{s}}+c_{\mathrm{b}}\right)}=\left(1-e^{z / N}\right)\left(1-e^{z / M}\right)
$$

we also have

$$
\Phi_{\mathrm{b}}\left(\frac{z}{2 \pi \mathrm{s}}+c_{\mathrm{b}}\right)=\frac{e^{\frac{\mathrm{i}}{2 \pi \mathrm{s}^{2}} \operatorname{Li}_{2}\left(e^{z}\right)}\left(1-e^{z}\right)^{1+\frac{\mathrm{i} z}{2 \pi \mathrm{s}^{2}}}}{\not_{N}\left(e^{z / N} ; q_{+}\right) \not D_{M}\left(e^{z / M} ; q_{-}\right)},
$$

Remark 1.10. The cyclic dilogarithm is in a sense a radial limit of the generating series

$$
M(x, q)=\prod_{n=1}^{\infty} \frac{1}{\left(1-x q^{n}\right)^{n}}
$$

where $M(x, q)$ is the McMahon generating series of 3-dimensional plane partitions; see [AKMV05] and [ORV06, Sec.2.1]. The latter appear in M-theory and mirror symmetry. It would be interesting and useful to understand a precise relation between the function $\mathrm{D}_{N}$ and plane partitions.

\section{Acknowledgments}

The paper originated as an attempt to prove an identity conjectured by joint work of Zagier and the first author. We wish to thank Tudor Dimofte and especially Don Zagier for enlightening conversations and for a generous sharing of their ideas. The paper was conceived during a conference in Vietnam in 2013, and largely completed in Geneva in 2014 at the Confucius Institute of the University of Geneva, and during a conference in Quantum Topology in Magnitogorsk, Russia in 2014 and during an Oberwolfach workshop in August 2014. The authors wish to thank the organizers of the conferences for their hospitality.

S.G. was supported in part by grant DMS-0805078 of the US National Science Foundation. R.K. was supported in part by the Swiss National Science Foundation. 


\section{Integrals of quasi-periodic functions}

\subsection{Some lemmas from complex analysis}

Lemma 2.1. Let $\mathrm{f}: \mathcal{U} \rightarrow \mathbb{C}$ be an analytic function satisfying the functional equation

$$
\mathrm{f}(z-a) \mathrm{f}(z+a)=\mathrm{f}(z)^{2}
$$

with some fixed $a \in \mathbb{C} \backslash\{0\}$, the domain $\mathcal{U} \subset \mathbb{C}$ being a translationally invariant open set, $\mathcal{U}=a+\mathcal{U}$, and $\mathcal{C} \subset \mathcal{U}$ an oriented path such that $\mathrm{f}(z)(\mathrm{f}(z)-$ $\mathrm{f}(z+a)) \neq 0$ for all $z \in \mathcal{C}$. Then

$$
\int_{\mathcal{C}} \mathrm{f}(z) \mathrm{d} z=\left(\int_{\mathcal{C}}-\int_{a+\mathcal{C}}\right) \frac{\mathrm{f}(z)}{1-\mathrm{f}(z+a) / \mathrm{f}(z)} \mathrm{d} z .
$$

Proof. We have

$$
\begin{aligned}
& \left(\int_{\mathcal{C}}-\int_{a+\mathcal{C}}\right) \frac{\mathrm{f}(z)}{1-\mathrm{f}(z+a) / \mathrm{f}(z)} \mathrm{d} z \\
= & \int_{\mathcal{C}} \frac{\mathrm{f}(z)}{1-\mathrm{f}(z+a) / \mathrm{f}(z)} \mathrm{d} z-\int_{a+\mathcal{C}} \frac{\mathrm{f}(z)}{1-\mathrm{f}(z) / \mathrm{f}(z-a)} \mathrm{d} z \\
= & \int_{\mathcal{C}} \frac{\mathrm{f}(z)-\mathrm{f}(z+a)}{1-\mathrm{f}(z+a) / \mathrm{f}(z)} \mathrm{d} z=\int_{\mathcal{C}} \mathrm{f}(z) \mathrm{d} z .
\end{aligned}
$$

Given a rational function $\mathrm{r}(z) \in \mathbb{C}(z)$, a nonzero complex number $q \in$ $\mathbb{C} \backslash\{0\}$ and an integer $k$ we define $\mathrm{r}_{k}(z ; q) \in \mathbb{C}(z), k \in \mathbb{Z}$, by:

$$
\frac{\mathrm{r}_{k+1}(z ; q)}{\mathrm{r}_{k}(z ; q)}=\mathrm{r}\left(z q^{k}\right), \quad \mathrm{r}_{0}(z ; q)=1
$$

Note that

$$
\mathrm{r}_{1}(z ; q)=\mathrm{r}(z)
$$

and

$$
\mathrm{r}_{k+l}(z ; q)=\mathrm{r}_{k}\left(z q^{l} ; q\right) \mathrm{r}_{l}(z ; q)=\mathrm{r}_{l}\left(z q^{k} ; q\right) \mathrm{r}_{k}(z ; q)
$$

for all integers $k, l$. In particular, in the case of roots of unity this implies a quasi-periodicity property:

$$
\mathrm{r}_{k+N}(z ; q)=\mathrm{r}_{k}(z ; q) \mathrm{r}_{N}(z ; q), \quad \text { if } q^{N}=1,
$$


and the invariance property

$$
\mathrm{r}_{N}(z q ; q)=\mathrm{r}_{N}(z ; q), \quad \text { if } q^{N}=1
$$

Let $(M, N)$ be an admissible pair, and recall b and $\mathrm{s}$ from Equation (11). Choose two integers $P$ and $Q$ which satisfy the equation $M P+N Q=1$. Let $\mathrm{f}(z)$ be a meromorphic function and $\mathrm{g}^{ \pm}(z) \in \mathbb{C}(z)$ two rational functions such that

$$
\frac{\mathrm{f}\left(z+\mathrm{b}^{ \pm 1} \mathbf{i}\right)}{\mathrm{f}(z)}=\mathrm{g}^{ \pm}\left(e^{2 \pi \mathrm{b}^{ \pm 1} z}\right)
$$

Then, for $k \in \mathbb{Z}$ we have

$$
\mathrm{g}^{ \pm}{ }_{k}\left(e^{2 \pi \mathrm{b}^{ \pm 1} z} ; q_{ \pm}\right)=\frac{\mathrm{f}\left(z+\mathrm{b}^{ \pm 1} \mathrm{i} k\right)}{\mathrm{f}(z)}
$$

and, in particular,

$$
\begin{aligned}
\mathrm{g}^{+}{ }_{N}\left(e^{2 \pi \mathrm{b} z} ; q_{+}\right) & =\frac{\mathrm{f}(z+\mathrm{bi} N)}{\mathrm{f}(z)}=\frac{\mathrm{f}(z+\mathrm{si})}{\mathrm{f}(z)}=\frac{\mathrm{f}\left(z+\mathrm{b}^{-1} \mathrm{i} M\right)}{\mathrm{f}(z)} \\
& =\mathrm{g}^{-}{ }_{M}\left(e^{2 \pi \mathrm{b}^{-1} z} ; q_{-}\right)
\end{aligned}
$$

Define

$$
\begin{aligned}
\mathrm{g}(x) & =\mathrm{g}^{+}{ }_{N}\left(x^{\frac{1}{N}} ; q_{+}\right)=\mathrm{g}^{-}{ }_{M}\left(x^{\frac{1}{M}} ; q_{-}\right) \\
\mathrm{S}(x, y) & =\sum_{k=0}^{\mathrm{s}^{2}-1} \mathrm{~g}^{+}{ }_{k P}\left(x ; q_{+}\right) \mathrm{g}^{-}{ }_{k Q}\left(y ; q_{-}\right)
\end{aligned}
$$

Lemma 2.2. (a) We have $\mathrm{g}(x) \in \mathbb{C}(x)$ and $\mathrm{S}(x, y) \in \mathbb{C}(x, y)$.

(b) The function $\mathrm{S}(x, y)$ is independent of $P$ and $Q$ provided $x^{N}=y^{M}$.

Proof. Since

$$
\mathrm{g}^{+}{ }_{N}\left(x q_{+} ; q_{+}\right)=\mathrm{g}^{+}{ }_{N}\left(x ; q_{+}\right), \quad \mathrm{g}_{M}^{-}\left(x q_{-} ; q_{-}\right)=\mathrm{g}_{M}^{-}\left(x ; q_{-}\right),
$$

it follows that $\mathrm{g}(x) \in \mathbb{C}(x)$, and consequently, $\mathrm{S}(x, y) \in \mathbb{C}(x, y)$.

For (b), let $P^{\prime}, Q^{\prime}$ be another pair satisfying the equation $M P^{\prime}+N Q^{\prime}=$ 1. Then there exists an integer $R$ such that $P^{\prime}=P+R N$ and $Q^{\prime}=Q-$ 
$R M$. Denoting the function (38) with $P$ and $Q$ replaced by $P^{\prime}$ and $Q^{\prime}$ as $\mathrm{S}^{\prime}(x, y)$, we have

$$
\begin{aligned}
\mathrm{S}^{\prime}(x, y) & =\sum_{k=0}^{\mathrm{s}^{2}-1} \mathrm{~g}^{+}{ }_{k P^{\prime}}\left(x ; q_{+}\right) \mathrm{g}^{-}{ }_{k Q^{\prime}}\left(y ; q_{-}\right) \\
& =\sum_{k=0}^{s^{2}-1} \mathrm{~g}^{+}{ }_{k P+k R N}\left(x ; q_{+}\right) \mathrm{g}^{-}{ }_{k Q-k R M}\left(y ; q_{-}\right) \\
& =\sum_{k=0}^{s^{2}-1} \mathrm{~g}^{+}{ }_{k P}\left(x ; q_{+}\right) \mathrm{g}^{+}{ }_{N}\left(x ; q_{+}\right)^{k R} \mathrm{~g}_{k Q}^{-}\left(y ; q_{-}\right) \mathrm{g}^{-}{ }_{M}\left(y ; q_{-}\right)^{-k R} \\
& =\sum_{k=0}^{s^{2}-1} \mathrm{~g}^{+}{ }_{k P}\left(x ; q_{+}\right)\left(\frac{\mathrm{g}^{+}{ }_{N}\left(x ; q_{+}\right)}{\mathrm{g}^{-}{ }_{M}\left(y ; q_{-}\right)}\right)^{k R} \mathrm{~g}^{-}{ }_{k Q}\left(y ; q_{-}\right) \\
& =\sum_{k=0}^{s^{2}-1} \mathrm{~g}^{+}{ }_{k P}\left(x ; q_{+}\right) \mathrm{g}^{-}{ }_{k Q}\left(y ; q_{-}\right) \\
& =\mathrm{S}(x, y)
\end{aligned}
$$

where we used the second equality in (37).

For a complex number $x$, we denote $\mathcal{C}_{x}=x \mathrm{i} / \mathrm{s}+\mathbb{R} \subset \mathbb{C}$.

Lemma 2.3. Let $\mathrm{f}(z), \mathrm{g}(z), \mathrm{S}(x, y)$ be as above and $\lambda$ a real number in general position such that the form $\mathrm{f}(z) \mathrm{d} z$ is absolutely integrable along $\mathcal{C}_{\lambda}$,

$$
\lim _{x \rightarrow \pm \infty} \sup _{y \in\left[\frac{\lambda}{s}, \frac{\lambda}{s}+\mathrm{s}\right]}|\mathrm{f}(x+\mathrm{i} y)|=0,
$$

and

$$
\mathrm{g}(0) \neq 1 \neq \mathrm{g}(\infty) .
$$

Then the following equalities hold

$$
\begin{aligned}
\int_{\mathcal{C}_{\lambda}} \mathrm{f}(z) \mathrm{d} z & =\left(\int_{\mathcal{C}_{\lambda}}-\int_{\mathcal{C}_{\lambda+1}}\right) \frac{\mathrm{f}(z) \mathrm{S}\left(e^{2 \pi \mathrm{b} z}, e^{2 \pi \mathrm{b}^{-1} z}\right)}{1-\mathrm{g}\left(e^{2 \pi \mathrm{s} z}\right)} \mathrm{d} z \\
& =2 \pi \mathrm{i} \sum_{0<\operatorname{sIm} \alpha-\lambda<1} \operatorname{Res}_{z=\alpha} \frac{\mathrm{f}(z) \mathrm{S}\left(e^{2 \pi \mathrm{b} z}, e^{2 \pi \mathrm{b}^{-1} z}\right)}{1-\mathrm{g}\left(e^{2 \pi \mathrm{s} z}\right)} .
\end{aligned}
$$


Proof. Let us derive the first equality. We denote

$$
q_{ \pm}=e^{2 \pi \mathrm{ib} \pm 2}
$$

so that we have

$$
q_{+}=e^{2 \pi \mathrm{i} \frac{M}{N}}, \quad q_{-}=e^{2 \pi \mathrm{i} \frac{N}{M}}
$$

We also have

$$
\mathrm{f}\left(z+k \mathbf{b}^{ \pm 1} \mathbf{i}\right)=\mathrm{f}(z) \mathrm{g}^{ \pm}{ }_{k}\left(e^{2 \pi \mathrm{b}^{ \pm 1} z} ; q_{ \pm}\right), \quad \forall k \in \mathbb{Z}
$$

In particular,

$$
\mathrm{f}(z+\mathrm{si})=\mathrm{f}(z+N \mathrm{bi})=\mathrm{f}\left(z+M \mathrm{~b}^{-1} \mathrm{i}\right)=\mathrm{f}(z) \mathrm{g}\left(e^{2 \pi \mathrm{s} z}\right)
$$

The function

$$
\mathrm{h}(z)=\mathrm{f}\left(\frac{z}{2 \pi \mathrm{s}}\right)
$$

has the properties

(48) $\quad \frac{\mathrm{h}(z+k 2 \pi \mathrm{i})}{\mathrm{h}(z)}=\mathrm{g}^{+}{ }_{P k}\left(e^{z / N} ; q_{+}\right) \mathrm{g}^{-}{ }_{Q k}\left(e^{z / M} ; q_{-}\right), \quad \forall k \in \mathbb{Z}$.

From Equation (48), it follows that

$$
\sum_{k=0}^{\mathrm{s}^{2}-1} \frac{\mathrm{h}(z+k 2 \pi \mathrm{i})}{\mathrm{h}(z)}=\mathrm{S}\left(e^{z / N}, e^{z / M}\right)
$$

As $\lambda$ is generic, the contour $\mathcal{C}_{\lambda}$ satisfies the conditions of Lemma 2.1 with $a=$ si. Thus, we write 
$(50)$

$$
\begin{aligned}
\int_{\mathcal{C}_{\lambda}} \mathrm{f}(z) \mathrm{d} z & =\left(\int_{\mathcal{C}_{\lambda}}-\int_{\mathrm{si}+\mathcal{C}_{\lambda}}\right) \frac{\mathrm{f}(z)}{1-\mathrm{g}\left(e^{2 \pi \mathrm{s} z}\right)} \mathrm{d} z \\
& =\left(\int_{\mathcal{C}_{\lambda 2 \pi \mathrm{s}}}-\int_{\mathcal{C}_{\left(\lambda+\mathrm{s}^{2}\right) 2 \pi \mathrm{s}}}\right) \frac{\mathrm{h}(z)}{1-\mathrm{g}\left(e^{z}\right)} \frac{\mathrm{d} z}{2 \pi \mathrm{s}} \\
& =\sum_{k=0}^{\mathrm{s}^{2}-1}\left(\int_{\mathcal{C}_{(\lambda+k) 2 \pi \mathrm{s}}}-\int_{\mathcal{C}_{(\lambda+k+1) 2 \pi \mathrm{s}}}\right) \frac{\mathrm{h}(z)}{1-\mathrm{g}\left(e^{z}\right)} \frac{\mathrm{d} z}{2 \pi \mathrm{s}} \\
& =\sum_{k=0}\left(\int_{\mathcal{C}_{\lambda 2 \pi \mathrm{s}}}-\int_{\mathcal{C}_{(\lambda+1) 2 \pi \mathrm{s}}}\right)^{2}-\frac{\mathrm{h}(z) \mathrm{S}\left(e^{z / N}, e^{z / M}\right)}{1-\mathrm{g}\left(e^{z}\right)} \frac{\mathrm{d} z}{2 \pi \mathrm{s}} \\
& =\left(\int_{\mathcal{C}_{\lambda 2 \pi \mathrm{s}}}-\int_{\mathcal{C}_{(\lambda+1) 2 \pi \mathrm{s}}} \frac{\mathrm{d} z}{2 \pi \mathrm{s}}\right. \\
& =\left(\int_{\mathcal{C}_{\lambda}}-\int_{\mathcal{C}_{\lambda+1}} \frac{\mathrm{f}(z) \mathrm{S}\left(e^{2 \pi \mathrm{b} z}, e^{2 \pi \mathrm{b}^{-1} z}\right)}{1-\mathrm{g}\left(e^{2 \pi \mathrm{s} z}\right)} \mathrm{d} z .\right.
\end{aligned}
$$

The second equality in Equation (42) follows from the residue theorem. To justify its application we first consider a countour integral along the boundary of a rectangle bound by $\mathcal{C}_{\lambda}-\mathcal{C}_{\lambda+1}$ and two vertical segments. Next, conditions (40), (41) imply that for sufficiently large negative or positive $x$ the integrand in the last part of (50) is regular on the vertical segment $\mathcal{C}^{\prime}=x+\mathrm{i}[\lambda, \lambda+1] / \mathrm{s}$ and the following estimate holds

$$
\begin{aligned}
& \left|\int_{\mathcal{C}^{\prime}} \frac{\mathrm{f}(z) \mathrm{S}\left(e^{2 \pi \mathrm{b} z}, e^{2 \pi \mathrm{b}^{-1} z}\right)}{1-\mathrm{g}\left(e^{2 \pi \mathrm{s} z}\right)} \mathrm{d} z\right| \\
& \leq \sum_{k=0}^{\mathrm{s}^{2}-1} \int_{\lambda}^{\lambda+1}\left|\frac{\mathrm{f}\left(x+\mathrm{i} \frac{y+k}{\mathrm{~s}}\right)}{1-\mathrm{g}\left(e^{2 \pi(\mathrm{s} x+\mathrm{i} y)}\right)}\right| \frac{\mathrm{d} y}{\mathrm{~s}} \leq \frac{1}{\mathrm{~s}} \sum_{k=0}^{\mathrm{s}^{2}-1} \sup _{y \in[\lambda, \lambda+1]}\left|\frac{\mathrm{f}\left(x+\mathrm{i} \frac{y+k}{\mathrm{~s}}\right)}{1-\mathrm{g}\left(e^{2 \pi(\mathrm{s} x+\mathrm{i} y)}\right)}\right| \\
& \leq \frac{1}{\mathrm{~s}} \sup _{y \in[\lambda, \lambda+1]} \frac{1}{\left|1-\mathrm{g}\left(e^{2 \pi(\mathrm{s} x+\mathrm{i} y)}\right)\right|} \sum_{k=0}^{\mathrm{s}^{2}-1} \sup _{t \in[\lambda, \lambda+1]}\left|\mathrm{f}\left(x+\mathrm{i} \frac{t+k}{\mathrm{~s}}\right)\right| \\
& \leq \mathrm{s} \frac{\sup _{z \in x+\mathrm{i}\left[\frac{\lambda}{\mathrm{s}}, \frac{\lambda}{\mathrm{s}}+\mathrm{s}\right]}|\mathrm{f}(z)|}{\inf _{|w|=e^{2 \pi s} \mid}|1-\mathrm{g}(w)|} \stackrel{|x| \rightarrow \infty}{\longrightarrow} 0
\end{aligned}
$$

where in the first inequality we use Formula (49) together with (47) and apply multiple times the triangle inequality for the absolute value and the integral. 


\subsection{Applications to 1-dimensional state-integrals: proof of Theorem 1.1}

In this section we prove Theorem 1.1. Fix integers $A$ and $B$ with $B>A>0$. The values of particular interest are $(A, B)=(1,2)$ and $(1,3)$ which correspond to the state-integrals of the knots $4_{1}$ and $5_{2}$ respectively. For the $4_{1}$ knot, see [AK14, Eqn.38] and [KLV12, Eqn.47] and [GK]. For the $5_{2}$ knot, see [KLV12, Eqn.53]. For the remaining values of $(A, B)$, although the 1dimensional state integral makes sense and it can be analyzed using our methods, there is no corresponding knot that we know of.

If

$$
\mathrm{f}\left(z-c_{\mathrm{b}}\right)=\Phi_{\mathrm{b}}(z)^{B} e^{-A \pi \mathrm{i} z^{2}}, \quad c_{\mathrm{b}}=\left(\mathrm{b}+\mathrm{b}^{-1}\right) \mathrm{i} / 2,
$$

then

$$
\begin{aligned}
\mathrm{g}(x) & =(-x)^{A}(1-x)^{-B} \\
\mathrm{~g}^{ \pm}(x) & =\mathrm{g}\left(q_{ \pm} x\right) \\
\mathrm{g}^{ \pm}{ }_{n}\left(x ; q_{ \pm}\right) & =(-x)^{A n} q_{ \pm}^{\frac{A}{2} n(n+1)}\left(q_{ \pm} x ; q_{ \pm}\right)_{n}^{-B}, \quad \forall n \in \mathbb{Z} .
\end{aligned}
$$

Observe that $\mathrm{f}(z)$ is non-vanishing and, due to the asymptotic behavior of the Faddeev's quantum dilogarithm given by (A.3), it is absolutely integrable along the line $\mathcal{C}_{\lambda}$ if

$$
-(M+N) / 2<\lambda<0,
$$

and $\mathrm{f}(z+\mathrm{si}) \neq \mathrm{f}(z)$ if $\lambda$ is in general position. Moreover, (53) and (A.3) imply the conditions (40). Indeed, for any $y \in\left[\frac{\lambda}{s}, \frac{\lambda}{s}+s\right]$, we have

$$
\begin{aligned}
& \lim _{x \rightarrow-\infty}|\mathrm{f}(x+\mathrm{i} y)| \\
= & \lim _{x \rightarrow-\infty}\left|\Phi_{\mathrm{b}}\left(x+\mathrm{i} y+c_{\mathbf{b}}\right)^{B} e^{-A \pi \mathrm{i}\left(x+\mathrm{i} y+c_{\mathrm{b}}\right)^{2}}\right|=\lim _{x \rightarrow-\infty}\left|e^{-A \pi \mathrm{i}\left(x+\mathrm{i} y+c_{\mathrm{b}}\right)^{2}}\right| \\
= & \lim _{x \rightarrow-\infty}\left|e^{-A \pi \mathrm{i} 2 x\left(\mathrm{i} y+c_{\mathrm{b}}\right)}\right|=\lim _{x \rightarrow-\infty}\left|e^{A \pi x\left(2 y+\mathrm{b}+\mathrm{b}^{-1}\right)}\right| \\
\leq & \lim _{x \rightarrow-\infty}\left|e^{A \pi x\left(2 \lambda \mathrm{s}^{-1}+\mathrm{b}+\mathrm{b}^{-1}\right)}\right|=\lim _{x \rightarrow-\infty}\left|e^{A \pi x \mathrm{~s}^{-1}(2 \lambda+M+N)}\right| \\
= & 0
\end{aligned}
$$


and

$$
\begin{aligned}
& \lim _{x \rightarrow+\infty}|\mathrm{f}(x+\mathrm{i} y)| \\
= & \lim _{x \rightarrow+\infty}\left|\Phi_{\mathrm{b}}\left(x+\mathrm{i} y+c_{\mathrm{b}}\right)^{B} e^{-A \pi \mathrm{i}\left(x+\mathrm{i} y+c_{\mathrm{b}}\right)^{2}}\right|=\lim _{x \rightarrow+\infty}\left|e^{(B-A) \pi \mathrm{i}\left(x+\mathrm{i} y+c_{\mathrm{b}}\right)^{2}}\right| \\
= & \lim _{x \rightarrow+\infty}\left|e^{(B-A) \pi \mathrm{i} 2 x\left(\mathrm{i} y+c_{\mathrm{b}}\right)}\right|=\lim _{x \rightarrow+\infty}\left|e^{-(B-A) \pi x\left(2 y+\mathrm{b}+\mathrm{b}^{-1}\right)}\right| \\
\leq & \lim _{x \rightarrow+\infty}\left|e^{-(B-A) \pi x\left(2 \lambda \mathrm{s}^{-1}+\mathrm{b}+\mathrm{b}^{-1}\right)}\right|=\lim _{x \rightarrow+\infty}\left|e^{-(B-A) \pi x \mathrm{~s}^{-1}(2 \lambda+M+N)}\right| \\
= & 0 .
\end{aligned}
$$

By using (25), we obtain that

$$
\begin{aligned}
\mathrm{f}(z) & =\Phi_{\mathrm{b}}\left(z+c_{\mathrm{b}}\right)^{B} e^{-A \pi \mathrm{i}\left(z+c_{\mathrm{b}}\right)^{2}} \\
& =\frac{e^{\frac{\mathrm{i} B}{2 \pi \mathrm{s}^{2}} \operatorname{Li}_{2}\left(e^{2 \pi \mathrm{s} z}\right)}\left(1-e^{2 \pi \mathrm{s} z}\right)^{B+B \mathrm{i} z \mathrm{~s}^{-1}}}{\not_{N}\left(e^{2 \pi \mathrm{b} z} ; q_{+}\right)^{B} \not D_{M}\left(e^{2 \pi \mathrm{b}^{-1} z} ; q_{-}\right)^{B}} e^{-A \pi \mathrm{i}\left(z+c_{\mathrm{b}}\right)^{2}} .
\end{aligned}
$$

By using the identity

$$
\begin{aligned}
e^{-A \pi \mathrm{i}\left(z+c_{\mathrm{b}}\right)^{2}} & =e^{-A \pi \mathrm{i}\left(c_{\mathrm{b}}+\frac{\mathrm{i}}{2 \mathrm{~s}}\right)^{2}} e^{A(2 \pi \mathrm{s} z-\pi \mathrm{i})\left(1-4 \mathrm{si} c_{\mathrm{b}}-2 \mathrm{si} z\right) \mathrm{s}^{-2} / 4} \\
& =e^{A \pi \mathrm{i} \frac{(M+N+1)^{2}}{4 M N}} e^{A(2 \pi \mathrm{s} z-\pi \mathrm{i})\left(1-4 \mathrm{si} c_{\mathrm{b}}-2 \mathrm{si} z\right) \mathrm{s}^{-2} / 4}
\end{aligned}
$$

and $(52 \mathrm{a})$, we can rewrite $(56)$ in the form

$$
\begin{aligned}
\mathrm{f}(z)= & \frac{e^{\frac{i B}{2 \pi \mathrm{s}^{2}} \mathrm{R}\left(e^{2 \pi \mathrm{s} z}\right)\left(1-e^{2 \pi \mathrm{s} z}\right)^{\frac{(2 N+1)(2 M+1)}{4 M N} B}}}{\not_{N}\left(e^{2 \pi \mathrm{b} z} ; q_{+}\right)^{B} \not D_{M}\left(e^{2 \pi \mathrm{b}^{-1} z} ; q_{-}\right)^{B}} \\
& \cdot e^{\pi \mathrm{i} \frac{B+3 A(M+N+1)^{2}}{12 M N}} \mathrm{~g}\left(e^{2 \pi \mathrm{s} z}\right)^{\frac{1-4 \mathrm{sic} \mathrm{b}_{-}-2 \mathrm{~s} z}{4 \mathrm{~s}^{2}}}
\end{aligned}
$$

where $R(x)$ is the Rogers dilogarithm (8).

It is easy to see that the only singularities in Equation (42) are simple poles that come from solutions to the equation $1-\mathrm{g}\left(e^{2 \pi \mathrm{s} z}\right)=1$. Moreover, if $z=\alpha$ is a solution with $0<\mathrm{s} \operatorname{Im} \alpha-\lambda<1$, then

$$
2 \pi \operatorname{Res}_{z=\alpha} \frac{\mathrm{f}(z) \mathrm{S}\left(e^{2 \pi \mathrm{b} z}, e^{2 \pi \mathrm{b}^{-1} z}\right)}{1-\mathrm{g}\left(e^{2 \pi \mathrm{s} z}\right)}=\mathrm{i}^{-1} \mathrm{~s}^{-1} \frac{\mathrm{f}(\alpha) \mathrm{S}\left(e^{2 \pi \mathrm{b} \alpha}, e^{2 \pi \mathrm{b}^{-1} \alpha}\right)}{e^{2 \pi \mathrm{s} \alpha} \mathrm{g}^{\prime}\left(e^{2 \pi \mathrm{s} \alpha}\right)} .
$$

Combining Lemma 2.3 with Equation (57) concludes the proof of Theorem 1.1. 


\subsection{The case of the $(-2,3,7)$ pretzel knot}

The (renormalized) Teichmüller TQFT partition function of the $(-2,3,7)$ pretzel knot, up to an overall phase factor, is the following 1-dimensional state integral, which we take as input to our analysis:

$$
I_{(-2,3,7)}(\mathrm{b})=\int_{\mathbb{R}+i \epsilon} \Phi_{\mathrm{b}}(x)^{2} \Phi_{\mathrm{b}}\left(2 x-c_{\mathrm{b}}\right) e^{-2 \pi \mathrm{i} x^{2}} d x,
$$

see Appendix B for details of derivation of this formula from the principles of the Teichmüller TQFT of [AK14]. The integral is absolutely convergent, and the statement and proof of Theorem 1.1 applies using the following definitions of the functions $\mathrm{f}(x), \mathrm{g}^{ \pm}{ }_{k}(x, q)$ and $\mathrm{g}(x)$ :

$$
\begin{aligned}
\mathrm{f}\left(x-c_{\mathrm{b}}\right) & =\Phi_{\mathrm{b}}(x)^{2} \Phi_{\mathrm{b}}\left(2 x-c_{\mathrm{b}}\right) e^{-2 \pi \mathrm{i} x^{2}} \\
\mathrm{~g}^{ \pm}{ }_{k}(x, q) & =\frac{q^{k(k+1)} x^{2 k}}{(q x ; q)_{k}^{2}\left(q x^{2} ; q\right)_{2 k}} \\
\mathrm{~g}(x) & =\frac{x^{2}}{(1-x)^{2}\left(1-x^{2}\right)^{2}} .
\end{aligned}
$$

Observe that $\mathrm{f}(z)$ is non-vanishing and absolutely integrable along the line $\mathcal{C}_{\lambda}$ if

$$
-(M+N) / 4<\lambda<0,
$$

and $\mathrm{f}(z+\mathrm{si}) \neq \mathrm{f}(z)$ if $\lambda$ is in general position.

We now discuss the solutions of the equation $\mathrm{g}(x)=1$ and the matching with the set of nonabelian parabolic $\operatorname{PSL}(2, \mathbb{C})$ representations, illustrating Remark 1.6.

The equation $\mathrm{g}(x)=1$ has 6 solutions that come from two cubic equations:

$$
\frac{z}{\left(1-z^{2}\right)(1-z)}= \pm 1
$$

Each triple of solutions lies in number fields $F_{+}$and $F_{-}$of discriminant -23 and 49 and type $[1,1]$ and $[3,0]$ respectively.

On the other hand, there are 6 nonabelian parabolic $\operatorname{PSL}(2, \mathbb{C})$ representations of the $(-2,3,7)$ pretzel knot. These may be found using the Ptolemy methods of [GGZ14] and their snappy implementation [CDW]. An 
alternative method is to use the $A$-polynomial of the pretzel knot from [Cul]

$$
\begin{aligned}
A(m, l)= & l^{6}-l^{5} m^{8}+2 l^{5} m^{9}-l^{5} m^{10}-2 l^{4} m^{18}-l^{4} m^{19} \\
& +l^{2} m^{36}+2 l^{2} m^{37}+l m^{45}-2 l m^{46}+l m^{47}-m^{55}
\end{aligned}
$$

Observe that $A(1, l)=(l-1)^{3}(l+1)^{3}$. Setting $(m, l)=\left(1+t, \pm 1+c_{ \pm} t+\right.$ $\left.O\left(t^{2}\right)\right)$ we obtain that

$$
\begin{aligned}
& -6119+2012 c_{-}-220 c_{-}^{2}+8 c_{-}^{3}=0 \\
& -6193-2020 c_{+}-220 c_{+}^{2}-8 c_{+}^{3}=0
\end{aligned}
$$

Then, we have $F_{ \pm}=\mathbb{Q}\left(c_{ \pm}\right)$. If $z$ is a solution to $(61)$, let $\rho_{z}$ denote the corresponding nonabelian parabolic $\operatorname{PSL}(2, \mathbb{C})$ representation. The Rogers dilogarithm of $z$ agrees with the complex volume of $\rho_{z}$, and $\mathrm{g}^{\prime}(x)$ agrees with the 1-loop invariant of $\rho_{z}$.

Incidentally, if $z \in F_{+}$, a totally real field, then the corresponding triple of elements of the Bloch group is torsion and triple of complex volumes is given by

$$
\left(\mathrm{e}\left(-\frac{19}{42}\right), \mathrm{e}\left(-\frac{13}{42}\right), \mathrm{e}\left(\frac{11}{42}\right)\right)=\mathrm{e}\left(-\frac{19}{42}\right)\left(1, \mathrm{e}\left(\frac{1}{7}\right), \mathrm{e}\left(-\frac{2}{7}\right)\right)
$$

where $\mathrm{e}(x)$ is given by Equation (20).

\section{Proof of Theorem 1.9}

We start by taking the logarithmic derivative of Faddeev's quantum dilogarithm

$$
\begin{aligned}
\frac{\partial}{\partial x} \log \Phi_{\mathrm{b}}(x) & =\int_{\mathbb{R}+\mathrm{i} \epsilon} \frac{-2 \mathrm{i} e^{-2 \mathrm{i} x z}}{4 \sinh (z \mathrm{~b}) \sinh \left(z \mathrm{~b}^{-1}\right)} \mathrm{d} z \\
& =\int_{\mathbb{R}+\mathrm{i} \epsilon} \frac{e^{-2 \mathrm{i} x z}}{2 \mathrm{i} \sinh (z \mathrm{~b}) \sinh \left(z \mathrm{~b}^{-1}\right)} \mathrm{d} z \\
& =\int_{\mathbb{R}+\mathrm{i} \epsilon} \frac{\pi \mathrm{s} e^{-2 \pi \mathrm{i} x z}}{2 \mathrm{i} \sinh (\pi z \mathrm{bs}) \sinh \left(\pi z \mathrm{~b}^{-1} \mathrm{~s}\right)} \mathrm{d} z \\
& =\int_{\mathbb{R}+\mathrm{i} \epsilon} \frac{\pi \mathrm{s} e^{-2 \pi \mathrm{i} x} z}{2 \mathrm{i} \sinh (\pi z M) \sinh (\pi z N)} \mathrm{d} z
\end{aligned}
$$


After rescaling $x \mapsto \frac{x}{2 \pi \mathrm{s}}$ we obtain

$$
4 \mathrm{i} \frac{\partial}{\partial x} \log \Phi_{\mathrm{b}}\left(\frac{x}{2 \pi \mathrm{s}}\right)=\int_{\mathbb{R}+\mathrm{i} \epsilon} \frac{e^{-\mathrm{i} x z}}{\sinh (\pi z M) \sinh (\pi z N)} \mathrm{d} z .
$$

The integrand in (63), given by the function

$$
\mathrm{f}(z)=\frac{e^{-\mathrm{i} x z}}{\sinh (\pi z M) \sinh (\pi z N)}
$$

satisfies Equation (26) with $a=\mathrm{i}$ as a direct consequence of the equalities

$$
\frac{\mathrm{f}(z \pm \mathrm{i})}{\mathrm{f}(z)}=(-1)^{M+N} e^{ \pm x}
$$

Equation (27) and an application of Cauchy's residue theorem implies that

$$
\begin{aligned}
& \frac{2}{\pi}\left(1-(-1)^{M+N} e^{x}\right) \frac{\partial}{\partial x} \log \Phi_{\mathrm{b}}\left(\frac{x}{2 \pi \mathrm{s}}\right) \\
= & \frac{1}{2 \pi \mathrm{i}}\left(\int_{\mathbb{R}+\mathrm{i} \epsilon}-\int_{\mathbb{R}+\mathrm{i}(1+\epsilon)}\right) \mathrm{f}(z) \mathrm{d} z \\
= & S_{1}(z)+S_{2}(z)+S_{3}(z),
\end{aligned}
$$

where

$$
S_{1}=\sum_{m=1}^{M-1} \operatorname{Res}_{z=\mathrm{i} \frac{m}{M}} \mathrm{f}(z), \quad S_{2}=\sum_{n=1}^{N-1} \operatorname{Res}_{z=\mathrm{i} \frac{n}{N}} \mathrm{f}(z), \quad S_{3}=\operatorname{Res}_{z=\mathrm{i}} \mathrm{f}(z) .
$$

So, we have reduced the integrals to the sum of residues. Our next task is to calculate each residue. Let us introduce $C_{i}$ for $i=1,2,3$ by:

$$
\begin{aligned}
C_{1} & =\frac{\left(1-e^{x+\pi \mathrm{i}(M+N)}\right)^{\frac{M-1}{2 M}}}{D_{M}\left(e^{(x+\pi \mathrm{i}(M+N)) / M} ; e^{2 \pi \mathrm{i} N / M}\right)} \\
C_{2} & =\frac{\left(1-e^{x+\pi \mathrm{i}(M+N)}\right)^{\frac{N-1}{2 N}}}{D_{N}\left(e^{(x+\pi \mathrm{i}(M+N)) / N} ; e^{2 \pi \mathrm{i} M / N}\right)} \\
C_{3} & =\left(1-(-1)^{M+N} e^{x}\right)^{\frac{\mathrm{i} x}{2 \pi \mathrm{s}^{2}}} e^{\frac{\mathrm{i}}{2 \pi \mathrm{s}^{2}} \operatorname{Li}_{2}\left((-1)^{M+N} e^{x}\right)} .
\end{aligned}
$$

Lemma 3.1. For $i=1,2,3$ we have:

$$
S_{i}=\frac{2}{\pi}\left(1-(-1)^{M+N} e^{x}\right) \frac{\partial}{\partial x} \log C_{i} .
$$


Proof. First we compute $S_{1}$. Expanding in powers of $z$ around $z=0$, we have

$$
\begin{aligned}
\mathrm{f}\left(z+\mathrm{i} \frac{m}{M}\right) & =\frac{(-1)^{m} e^{m x / M}}{\pi z M \mathrm{i} \sin (\pi m N / M)}(1+\mathrm{O}(z)) \\
& =\frac{(-1)^{m} e^{m x / M}}{\pi z M \mathrm{i} \sin (\pi m N / M)}+\mathrm{O}(1)
\end{aligned}
$$

so that

$$
\begin{aligned}
\operatorname{Res}_{z=\mathrm{i} \frac{m}{M}} \mathrm{f}(z) & =\frac{\left(-e^{x / M}\right)^{m}}{\pi \mathrm{i} M \sin (\pi m N / M)}=\frac{2\left(-e^{x / M}\right)^{m}}{\pi M\left(e^{\pi \mathrm{i} m N / M}-e^{-\pi \mathrm{i} m N / M}\right)} \\
& =\frac{-2\left(-e^{(x+\pi \mathrm{i} N) / M}\right)^{m}}{\pi M\left(1-e^{2 \pi \mathrm{i} m N / M}\right)}=\frac{-2 e^{m(x+\pi \mathrm{i}(M+N)) / M}}{\pi M\left(1-e^{2 \pi \mathrm{i} m N / M}\right)} .
\end{aligned}
$$

Now, by using Lemma 3.2 (see below), we calculate

$$
\begin{aligned}
-\frac{\pi}{2} \sum_{m=1}^{M-1} \operatorname{Res}_{z=\mathrm{i} \frac{m}{M}} \mathrm{f}(z)= & M^{-1} \sum_{m=1}^{M-1} \frac{e^{m(x+\pi \mathrm{i}(M+N)) / M}}{1-e^{2 \pi \mathrm{i} m N / M}} \\
= & \frac{M-1}{2 M} e^{x+\pi \mathrm{i}(M+N)}+\left(1-e^{x+\pi \mathrm{i}(M+N)}\right) \\
& \cdot \frac{\partial}{\partial x} \log D_{M}\left(e^{(x+\pi \mathrm{i}(M+N)) / M} ; e^{2 \pi \mathrm{i} N / M}\right) .
\end{aligned}
$$

Finally observe that

$$
-\int_{-\infty}^{x} \frac{e^{y+\pi \mathrm{i}(M+N)}}{\left(1-e^{y+\pi \mathrm{i}(M+N)}\right)} \mathrm{d} y=\log \left(1-e^{x+\pi \mathrm{i}(M+N)}\right) .
$$

This proves Equation (67) for $i=1$. Interchanging $M$ with $N$ proves Equation (67) for $i=2$. Finally we compute $S_{3}$. Expanding in powers of $z$ around $z=0$, we have

$$
\begin{aligned}
(-1)^{M+N} e^{-x} \mathrm{f}(z+\mathrm{i}) & =\mathrm{f}(z)=\frac{1-\mathrm{i} x z+\mathrm{O}\left(z^{2}\right)}{\pi z M\left(1+\mathrm{O}\left(z^{2}\right)\right) \pi z N\left(1+\mathrm{O}\left(z^{2}\right)\right)} \\
& =\frac{1-\mathrm{i} x z+\mathrm{O}\left(z^{2}\right)}{\pi^{2} M N z^{2}}=\frac{1}{\pi^{2} \mathrm{~s}^{2} z^{2}}-\frac{\mathrm{i} x}{\pi^{2} \mathrm{~s}^{2} z}+\mathrm{O}(1)
\end{aligned}
$$

so that

$$
\operatorname{Res}_{z=\mathrm{i}} \mathrm{f}(z)=\frac{(-1)^{1+M+N} \mathrm{i} x e^{x}}{\pi^{2} \mathrm{~s}^{2}} .
$$


Now we calculate

$$
\begin{aligned}
2 \pi \text { is }^{2} \log C_{3}= & \int_{-\infty}^{x} \frac{(-1)^{M+N} y e^{y} \mathrm{~d} y}{1-(-1)^{M+N} e^{y}}=-\int_{-\infty}^{x} y \mathrm{~d} \log \left(1-(-1)^{M+N} e^{y}\right) \\
= & -\left[y \log \left(1-(-1)^{M+N} e^{y}\right)\right]_{-\infty}^{x} \\
& +\int_{-\infty}^{x} \log \left(1-(-1)^{M+N} e^{y}\right) \mathrm{d} y \\
= & -x \log \left(1-(-1)^{M+N} e^{x}\right)+\int_{0}^{(-1)^{M+N} e^{x}} \frac{\log (1-z)}{z} \mathrm{~d} z \\
= & -x \log \left(1-(-1)^{M+N} e^{x}\right)-\operatorname{Li}_{2}\left((-1)^{M+N} e^{x}\right) .
\end{aligned}
$$

Equation (67) follows for $i=3$.

We now finish the proof of Theorem 1.9. Using

$$
\lim _{x \rightarrow-\infty} \Phi_{\mathrm{b}}(x)=1
$$

it follows that

$$
\log \Phi_{\mathrm{b}}\left(\frac{x}{2 \pi \mathrm{s}}\right)=\int_{-\infty}^{x} \frac{\partial}{\partial y} \log \Phi_{\mathrm{b}}\left(\frac{y}{2 \pi \mathrm{s}}\right) \mathrm{d} y
$$

Combining the above with Equation (66) and Lemma 3.1, we obtain that

$$
\Phi_{\mathrm{b}}\left(\frac{x}{2 \pi \mathrm{s}}\right)=C_{1} C_{2} C_{3} .
$$

Introduce a new variable $z$ related to $x$ by

$$
\frac{x}{2 \pi \mathrm{s}}=\frac{z}{2 \pi \mathrm{s}}-c_{\mathrm{b}} .
$$

In other words, we have

$$
x=z-\pi \mathrm{i}(M+N) .
$$

Equation (70) implies that

$$
\begin{aligned}
& \Phi_{\mathrm{b}}\left(\frac{z}{2 \pi \mathrm{s}}-c_{\mathrm{b}}\right) \mathrm{D}_{N}\left(e^{z / N} ; q_{+}\right) \mathrm{D}_{M}\left(e^{z / M} ; q_{-}\right) e^{-\frac{\mathrm{i}}{2 \pi \mathrm{s}^{2}} \operatorname{Li}_{2}\left(e^{z}\right)} \\
= & \left(1-e^{z}\right)^{\frac{M-1}{2 M}+\frac{N-1}{2 N}+\frac{\mathrm{i}(z-\pi \mathrm{i}(M+N))}{2 \pi M N}}=\left(1-e^{z}\right)^{1+\frac{\mathrm{i} z}{2 \pi M N}} .
\end{aligned}
$$

This concludes the proof of Theorem 1.9. 
Lemma 3.2. For any complex root of unity $q$ of order $M$, we have

$$
\sum_{m=1}^{M-1} \frac{x^{m}}{1-q^{m}}=\frac{M-1}{2} x^{M}+\left(1-x^{M}\right) x \frac{\partial}{\partial x} \log D_{M}(x ; q) .
$$

Proof. We calculate

$$
\begin{aligned}
& \left(1-x^{M}\right) x \frac{\partial}{\partial x} \log D_{M}(x ; q) \\
= & \left(1-x^{M}\right) x \frac{\partial}{\partial x} \sum_{m=1}^{M-1} \frac{m}{M} \log \left(1-x q^{m}\right) \\
= & -\frac{\left(1-x^{M}\right) x}{M} \sum_{m=1}^{M-1} \frac{m q^{m}}{1-x q^{m}}=-\frac{x}{M} \sum_{m=1}^{M-1} \frac{m q^{m}\left(1-\left(x q^{m}\right)^{M}\right)}{1-x q^{m}} \\
= & -\frac{x}{M} \sum_{m=1}^{M-1} m q^{m} \sum_{n=0}^{M-1}\left(x q^{m}\right)^{n}=-\frac{1}{M} \sum_{n=0}^{M-1} x^{n+1} \sum_{m=1}^{M-1} m q^{m(n+1)} \\
= & -\frac{1}{M} \sum_{n=1}^{M} x^{n} \sum_{m=1}^{M-1} m q^{m n}=-\frac{M-1}{2} x^{M}-\frac{1}{M} \sum_{n=1}^{M-1} x^{n} \sum_{m=1}^{M-1} m q^{m n} .
\end{aligned}
$$

To finish the proof, we do the final calculation

$$
\begin{aligned}
\sum_{m=1}^{M-1} m q^{m n} & =\left.t \frac{\partial}{\partial t} \sum_{m=1}^{M-1} t^{m}\right|_{t=q^{n}}=\left.t \frac{\partial}{\partial t}\left(\frac{1-t^{M}}{1-t}-1\right)\right|_{t=q^{n}} \\
& =\left.\frac{-M t^{M}}{1-t}\right|_{t=q^{n}}=\frac{-M}{1-q^{n}}
\end{aligned}
$$

\section{Appendix A. Some useful properties of the quantum dilogarithm}

The quantum dilogarithm $\Phi_{\mathrm{b}}(x)$ is defined by

$$
\Phi_{\mathrm{b}}(x)=\frac{\left(e^{2 \pi \mathrm{b}\left(x+c_{\mathrm{b}}\right)} ; q\right)_{\infty}}{\left(e^{2 \pi \mathrm{b}^{-1}\left(x-c_{\mathrm{b}}\right)} ; \tilde{q}\right)_{\infty}}
$$

where

$$
q=e^{2 \pi \mathrm{i}^{2}}, \quad \tilde{q}=e^{-2 \pi \mathrm{i}^{-2}}, \quad c_{\mathrm{b}}=\frac{\mathrm{i}}{2}\left(\mathrm{~b}+\mathrm{b}^{-1}\right), \quad \operatorname{Im}\left(\mathrm{b}^{2}\right)>0 .
$$


An integral representation is given by [Fad95]

$$
\Phi_{\mathrm{b}}(x)=\exp \left(\int_{\mathbb{R}+\mathrm{i} \epsilon} \frac{e^{-2 \mathrm{i} x z}}{4 \sinh (z \mathrm{~b}) \sinh \left(z \mathrm{~b}^{-1}\right)} \frac{\mathrm{d} z}{z}\right)
$$

in the strip $|\operatorname{Im} z|<\left|\operatorname{Im} c_{\mathrm{b}}\right|$. Remarkably, this function admits an extension to all values of $\mathrm{b}$ with $\mathrm{b}^{2} \notin \mathbb{R}_{\leq 0}$. $\Phi_{\mathrm{b}}(x)$ is a meromorphic function of $x$ with

$$
\text { poles: } c_{\mathrm{b}}+\mathrm{iNb}+\mathrm{iNb}^{-1}, \quad \text { zeros: }-c_{\mathrm{b}}-\mathrm{iNb}-\mathrm{iNb}^{-1} \text {. }
$$

The inversion relation

$$
\Phi_{\mathrm{b}}(x) \Phi_{\mathrm{b}}(-x)=e^{\pi \mathrm{i} x^{2}} \Phi_{\mathrm{b}}(0)^{2}, \quad \Phi_{\mathrm{b}}(0)=\left(\frac{q}{\tilde{q}}\right)^{\frac{1}{48}}=e^{\pi \mathrm{i}\left(\mathrm{b}^{2}+\mathrm{b}^{-2}\right) / 24}
$$

allows one to move $\Phi_{\mathrm{b}}(x)$ from the denominator to the numerator of the integrand of a state-integral.

The asymptotics of the quantum dilogarithm are given by [AK14, App.A]

$$
\Phi_{\mathrm{b}}(x) \sim\left\{\begin{array}{lll}
\Phi_{\mathrm{b}}(0)^{2} e^{\pi \mathrm{i} x^{2}} & \text { when } & \Re(x) \gg 0 \\
1 & \text { when } & \Re(x) \ll 0
\end{array} .\right.
$$

The quantum dilogarithm is a quasi-periodic function. Explicitly, it satisfies the equations

$$
\begin{aligned}
\frac{\Phi_{\mathrm{b}}\left(x+c_{\mathrm{b}}+\mathrm{ib}\right)}{\Phi_{\mathrm{b}}\left(x+c_{\mathrm{b}}\right)} & =\frac{1}{1-q e^{2 \pi \mathrm{b} x}} \\
\frac{\Phi_{\mathrm{b}}\left(x+c_{\mathrm{b}}+\mathrm{ib}^{-1}\right)}{\Phi_{\mathrm{b}}\left(x+c_{\mathrm{b}}\right)} & =\frac{1}{1-\tilde{q}^{-1} e^{2 \pi \mathrm{b}^{-1} x}} .
\end{aligned}
$$

\section{Appendix B. The Teichmüller TQFT partition function of the $(-2,3,7)$ pretzel knot}

In this section we calculate the (renormalized) partition function of the $(-2,3,7)$ pretzel knot following the definition of [AK14].

A 1-vertex H-triangulation of a knot in $S^{3}$ can be found from a knot diagram by using the same line of reasoning as in the case of ideal triangulations of knot complements, the only difference being that instead of contracting the whole knot to a point one does so only the complementary part to a chosen segment. 
For any integer $n$, we denote $[n]=\{k \in \mathbb{Z} \mid 0 \leq k \leq n\}$, and for an $n$ simplex $s$ of a delta-complex we write

$$
s \equiv\left(s \mid \partial_{0} s, \partial_{1} s, \ldots, \partial_{n} s\right)
$$

in order to indicate the information about the action of the boundary maps. If $D$ is a delta-triangulation, then we denote by $D_{i}$ the set of $i$-dimensional simplexes of $D$. With this notation, in the case of the $(-2,3,7)$ pretzel knot, we start with a 1-vertex $\mathrm{H}$-triangulation $X$ composed of one element vertex set $X_{0} \equiv\{*\}$, six element set of oriented edges $X_{1} \equiv\left\{e_{i}\right\}_{i \in[5]}$, ten element set of triangular faces $X_{2} \equiv\left\{f_{i}\right\}_{i \in[9]}$,

$$
\begin{aligned}
& \left(f_{0} \mid e_{1}, e_{1}, e_{2}\right),\left(f_{1} \mid e_{1}, e_{0}, e_{3}\right),\left(f_{2} \mid e_{2}, e_{3}, e_{3}\right),\left(f_{3} \mid e_{4}, e_{5}, e_{2}\right), \\
& \left(f_{4} \mid e_{4}, e_{1}, e_{3}\right),\left(f_{5} \mid e_{5}, e_{1}, e_{3}\right),\left(f_{6} \mid e_{5}, e_{3}, e_{4}\right),\left(f_{7} \mid e_{5}, e_{4}, e_{5}\right), \\
& \left(f_{8} \mid e_{3}, e_{4}, e_{2}\right),\left(f_{9} \mid e_{4}, e_{1}, e_{4}\right),
\end{aligned}
$$

and five element set of oriented tetrahedra $X_{3} \equiv\left\{t_{i}\right\}_{i \in[4]}$,

$$
\begin{aligned}
& \left(t_{0} \mid f_{0}, f_{1}, f_{1}, f_{2}\right),\left(t_{1} \mid f_{3}, f_{4}, f_{5}, f_{2}\right),\left(t_{2} \mid f_{6}, f_{7}, f_{8}, f_{3}\right), \\
& \left(t_{3} \mid f_{7}, f_{5}, f_{9}, f_{6}\right),\left(t_{4} \mid f_{4}, f_{9}, f_{0}, f_{8}\right),
\end{aligned}
$$

$t_{0}$ being negatively and all others positively oriented. The distinguished edge representing the knot is given by the edge $e_{0}$.

Following the rules of the Teichmüller TQFT of [AK14], we assume that triangulation $X$ is provided with a shape structure, i.e. each tetrahedron $t_{i}$ carries dihedral angles of an ideal hyperbolic tetrahedron. The gauge equivalence class of the shape structure of $X$ (with respect to the Neumann-Zagier symplectic structure) is characterized by the total angles around the (geometrical) edges $e_{i}$ :

$$
\begin{aligned}
& w\left(e_{0}\right)=\gamma_{0} \\
& w\left(e_{1}\right)=2 \pi-\gamma_{0}+\gamma_{1}+\gamma_{3}-\alpha_{4} \\
& w\left(e_{2}\right)=\gamma_{0}+\gamma_{1}+\alpha_{2}+\alpha_{4} \\
& w\left(e_{3}\right)=2 \pi-\gamma_{0}-\gamma_{1}+\beta_{2}+\beta_{3}+\gamma_{4} \\
& w\left(e_{4}\right)=2 \pi+\alpha_{1}+2 \gamma_{2}-\gamma_{3}-\gamma_{4} \\
& w\left(e_{5}\right)=2 \pi+\beta_{1}-\gamma_{2}-\beta_{3}
\end{aligned}
$$

where $\alpha_{i}, \beta_{i}$ and $\gamma_{i}$ denote the (dihedral) angles along the edges 01, 02 and 03 respectively of the tetrahedron $t_{i}$. The total angles are constrained by 
the following sum rule:

$$
\sum_{i \in[5]} w\left(e_{i}\right)=10 \pi
$$

which is easily verified by using (B.8).

The Teichmüller TQFT partition function $Z(X)$, up to an overall complex phase, can be written in the form of a multiple integral

$$
\begin{aligned}
Z(X) & =\int_{\mathbb{R}^{X_{2}}} \mathrm{~d} x \int_{\mathbb{R}^{[4]}} \mathrm{d} y \prod_{i \in[4]} M\left(t_{i},\left.x\right|_{\left(t_{i}\right)_{2}}, y_{i}\right) \phi\left(t_{i}, y_{i}\right) \\
& =\int_{\mathbb{R}^{[4]}} \mathrm{d} y K(X, y) \prod_{i \in[4]} \phi\left(t_{i}, y_{i}\right),
\end{aligned}
$$

where

$$
K(X, y) \equiv \int_{\mathbb{R}^{X_{2}}} \mathrm{~d} x \prod_{i \in[4]} M\left(t_{i},\left.x\right|_{\left(t_{i}\right)_{2}}, y_{i}\right)
$$

and where to a shaped tetrahedron $t \in X_{3}$ of $\operatorname{sign} \varepsilon \in\{ \pm 1\}$ and dihedral angles $\alpha_{i}$ along the edges connecting 0 -th with $i$-th vertices with $i \in\{1,2,3\}$, a map $x \in \mathbb{R}^{(t)_{2}}$ associating real numbers $x_{i}=x\left(\partial_{i} t\right)$ to the facets of $t$, and a real number $y \in \mathbb{R}$, we associate a tempered distribution

$$
\begin{aligned}
M(t, x, y) & =Q_{\varepsilon}\left(x_{0}, x_{1}, x_{2}, x_{3}, y\right) \\
& \equiv e^{2 \pi \mathrm{i} \varepsilon x_{0} y} \delta\left(x_{0}-x_{1}+x_{2}\right) \delta\left(x_{2}-x_{3}+y\right)
\end{aligned}
$$

and a function

$$
\phi(t, y)=\psi_{\varepsilon}\left(\alpha_{3}, \alpha_{2}, y\right) \equiv e^{-2 \mathrm{i} c_{\mathrm{b}} \alpha_{3} y} \Phi_{\mathrm{b}}\left(y-\varepsilon c_{\mathrm{b}} \frac{\alpha_{2}+\alpha_{3}}{\pi}\right)^{-\varepsilon}
$$

Compared to the definition given in [AK14], we inserted in (B.10) artificially extra integrations over the variables $y$ at the expense of equal number of extra delta-functions. 
Theorem B.1. Let the shape structure of $X$ satisfy the conditions

$$
\begin{array}{ll}
w\left(e_{0}\right)=(1-\lambda) \pi, & w\left(e_{1}\right)=w\left(e_{3}\right)=(1+\lambda) \pi, \\
w\left(e_{2}\right)=(3-\lambda) \pi, & w\left(e_{4}\right)=w\left(e_{5}\right)=2 \pi
\end{array}
$$

where $\lambda \in]-1,1[$. Then, up to an overall phase factor, the Teichmüller TQFT partition function $Z(X)$ defined in (B.10) is given by the formula

$$
Z(X)=\Phi_{\mathrm{b}}\left(\lambda c_{\mathrm{b}}\right) \int_{\mathbb{R}+\mathrm{i} \epsilon} \frac{\Phi_{\mathrm{b}}(z)^{2}}{\Phi_{\mathrm{b}}\left(c_{\mathrm{b}}-2 z\right)} \mathrm{d} z
$$

where $\epsilon$ is a small positive real number.

\section{B.1. Calculation of $K(X, y)$}

We start by calculating the function $K(X, y)$ defined in (B.11). Its structure is distinguished by the fact that the number of integrations coincides with the number of delta-functions, so that the result is a tempered distribution given by an oscillating exponential of a quadratic form in components of $y$.

Lemma B.2. The function $K(X, y)$ defined in (B.11) is given by the following formula:

$$
K(X, y)=e^{2 \pi \mathrm{i}\left(y_{1}\left(y_{2}+y_{4}\right)-\left(y_{2}+y_{3}+y_{4}\right)\left(y_{2}+2 y_{4}\right)\right)} .
$$

Proof. To keep the formulas compact, we introduce the following notation

$$
\begin{aligned}
& Q_{u, v}^{x, y, z}=Q_{+1}(x, u, y, v, z), \\
& \bar{Q}_{x, y, z}^{u, v}=Q_{-1}(x, u, y, v, z), \quad\langle x ; y\rangle=e^{2 \pi \mathrm{i} x y} .
\end{aligned}
$$

We have

$$
\begin{aligned}
K(X, y)= & \int_{\mathbb{R}^{[9]}} \mathrm{d} x \bar{Q}_{x_{0}, x_{1}, y_{0}}^{x_{1}, x_{2}} Q_{x_{4}, x_{2}}^{x_{3}, x_{5}, y_{1}} Q_{x_{7}, x_{3}}^{x_{6}, x_{8}, y_{2}} Q_{x_{5}, x_{6}}^{x_{7}, x_{9}, y_{3}} Q_{x_{9}, x_{8}}^{x_{4}, x_{0}, y_{4}} \\
= & \int_{\mathbb{R}^{10}} \mathrm{~d} x_{0} \cdots \mathrm{d} x_{9}\left\langle x_{0} ; y_{0}\right\rangle^{-1} \delta\left(x_{0}\right) \delta\left(x_{1}-x_{2}+y_{0}\right) \\
& \cdot Q_{x_{4}, x_{2}}^{x_{3}, x_{5}, y_{1}} Q_{x_{7}, x_{3}}^{x_{6}, x_{8}, y_{2}} Q_{x_{5}, x_{6}}^{x_{7}, x_{9}, y_{3}} Q_{x_{9}, x_{8}}^{x_{4}, x_{0}, y_{4}} \\
= & \int_{\mathbb{R}^{8}} \mathrm{~d} x_{2} \cdots \mathrm{d} x_{9} Q_{x_{4}, x_{2}, x_{1}}^{x_{3}, x_{5}, y_{1}} Q_{x_{7}, x_{3}}^{x_{6}, x_{8}, y_{2}} Q_{x_{5}, x_{6}}^{x_{7}, x_{9}, y_{3}} Q_{x_{9}, x_{8}}^{x_{4}, 0, y_{4}}
\end{aligned}
$$

where we used the explicit form of $\bar{Q}_{x_{0}, x_{1}, y_{0}}^{x_{1}, x_{2}}$ in order to integrate out the variables $x_{0}$ and $x_{1}$. Next, we use $Q_{x_{9}, x_{8}}^{x_{4}, 0, y_{4}}$ in order to integrate out the 
variables $x_{8}$ and $x_{9}$. We obtain that

$$
\begin{aligned}
K(X, y)= & \int_{\mathbb{R}^{8}} \mathrm{~d} x_{2} \cdots \mathrm{d} x_{9}\left\langle x_{4} ; y_{4}\right\rangle \delta\left(x_{4}-x_{9}\right) \delta\left(y_{4}-x_{8}\right) \\
& \cdot Q_{x_{4}, x_{2}}^{x_{3}, x_{5}, y_{1}} Q_{x_{7}, x_{3}}^{x_{6}, x_{8}, y_{2}} Q_{x_{5}, x_{6}}^{x_{7}, x_{9}, y_{3}} \\
= & \int_{\mathbb{R}^{6}} \mathrm{~d} x_{2} \cdots \mathrm{d} x_{7}\left\langle x_{4} ; y_{4}\right\rangle Q_{x_{4}, x_{2}}^{x_{3}, x_{5}, y_{1}} Q_{x_{7}, x_{3}}^{x_{6}, y_{4}, y_{2}} Q_{x_{5}, x_{6}}^{x_{7}, x_{4}, y_{3}}
\end{aligned}
$$

Then using $Q_{x_{5}, x_{6}}^{x_{7}, x_{4}, y_{3}}$ to integrate out the variables $x_{6}$ and $x_{7}$, we obtain

$$
\begin{aligned}
K(X, y)= & \int_{\mathbb{R}^{6}} \mathrm{~d} x_{2} \cdots \mathrm{d} x_{7}\left\langle x_{4} ; y_{4}\right\rangle\left\langle x_{7} ; y_{3}\right\rangle \\
& \cdot \delta\left(x_{7}-x_{5}+x_{4}\right) \delta\left(x_{4}-x_{6}+y_{3}\right) Q_{x_{4}, x_{2}}^{x_{3}, x_{5}, y_{1}} Q_{x_{7}, x_{3}}^{x_{6}, y_{4}, y_{2}} \\
= & \int_{\mathbb{R}^{4}} \mathrm{~d} x_{2} \cdots \mathrm{d} x_{5} \frac{\left\langle x_{5} ; y_{3}\right\rangle}{\left\langle x_{4} ; y_{3}-y_{4}\right\rangle} Q_{x_{4}, x_{2}}^{x_{3}, x_{5}, y_{1}} Q_{x_{5}-x_{4}, x_{3}}^{x_{4}+y_{3}, y_{4}, y_{2}} .
\end{aligned}
$$

Using $Q_{x_{4}, x_{2}}^{x_{3}, x_{5}, y_{1}}$ to integrate out $x_{2}$ and $x_{5}$, it follows that

$$
\begin{aligned}
K(X, y)= & \int_{\mathbb{R}^{4}} \mathrm{~d} x_{2} \cdots \mathrm{d} x_{5} \frac{\left\langle x_{5} ; y_{3}\right\rangle\left\langle x_{3} ; y_{1}\right\rangle}{\left\langle x_{4} ; y_{3}-y_{4}\right\rangle} \\
& \cdot \delta\left(x_{3}-x_{4}+x_{5}\right) \delta\left(x_{5}-x_{2}+y_{1}\right) Q_{x_{5}-x_{4}, x_{3}}^{x_{4}+y_{3}, y_{2}} \\
= & \int_{\mathbb{R}^{2}} \mathrm{~d} x_{3} \mathrm{~d} x_{4} \frac{\left\langle x_{4} ; y_{4}\right\rangle}{\left\langle x_{3} ; y_{3}-y_{1}\right\rangle} Q_{-x_{3}, x_{3}}^{x_{4}+y_{3}, y_{4}, y_{2}} .
\end{aligned}
$$

We finish the calculation by integrating out the remaining two varibles $x_{3}$ and $x_{4}$, to obtain that

$$
\begin{aligned}
K(X, y)= & \int_{\mathbb{R}^{2}} \mathrm{~d} x_{3} \mathrm{~d} x_{4} \frac{\left\langle x_{4} ; y_{4}\right\rangle\left\langle x_{4}+y_{3} ; y_{2}\right\rangle}{\left\langle x_{3} ; y_{3}-y_{1}\right\rangle} \\
& \cdot \delta\left(x_{4}+y_{3}+x_{3}+y_{4}\right) \delta\left(y_{4}-x_{3}+y_{2}\right) \\
= & \frac{\left\langle y_{1} ; y_{2}+y_{4}\right\rangle}{\left\langle y_{2}+y_{3}+y_{4} ; y_{2}+2 y_{4}\right\rangle} .
\end{aligned}
$$

\section{B.2. Proof of Theorem B.1}

In what follows, we will use the symbol $\simeq$ to denote an equality up to a phase depending on angle variables but independent of integration variables. 
Due to the fact that $K(X, y)$ does not depend on $y_{0}$, the partition function (B.10) takes the factorized form

$$
Z(X)=\tilde{Z}(X) \int_{\mathbb{R}} \mathrm{d} y_{0} \psi_{-}\left(\gamma_{0}, \beta_{0}, y_{0}\right) \simeq \tilde{Z}(X) \Phi_{\mathrm{b}}\left(c_{\mathrm{b}} \frac{\pi-\gamma_{0}}{\pi}\right)
$$

where

$$
\tilde{Z}(X)=\int_{\mathbb{R}^{4}} \mathrm{~d} y_{1} \cdots \mathrm{d} y_{4} K(X, y) \prod_{i=1}^{4} \psi_{+}\left(\gamma_{i}, \beta_{i}, y_{i}\right)
$$

is the renormalized partition function which becomes a knot invariant in the fully balanced case corresponding to the following choice of the total angles:

$$
w\left(e_{0}\right)=0 \quad \text { and } \quad w\left(e_{i}\right)=2 \pi, \forall i \in\{1, \ldots, 5\}
$$

which corresponds to $\lambda=1$ in (B.14). As this choice is singular for $Z(X)$ though non-singular for $\tilde{Z}(X)$, we proceed at this stage without imposing any restrictions on the shape structure.

By shortening the notation as

$$
\psi_{\alpha, \beta}(x) \equiv \psi_{+}(\alpha, \beta, x)
$$

we transform the renormalized partition function $\tilde{Z}(X)$ as follows:

$$
\begin{aligned}
\tilde{Z}(X)= & \int_{\mathbb{R}^{4}} \mathrm{~d} y_{1} \cdots \mathrm{d} y_{4} e^{2 \pi \mathrm{i}\left(y_{1}\left(y_{2}+y_{4}\right)-\left(y_{2}+y_{3}+y_{4}\right)\left(y_{2}+2 y_{4}\right)\right)} \prod_{i=1}^{4} \psi_{\gamma_{i}, \beta_{i}}\left(y_{i}\right) \\
= & \int_{\mathbb{R}^{2}} \mathrm{~d} y_{2} \mathrm{~d} y_{4} e^{-2 \pi \mathrm{i}\left(y_{2}+y_{4}\right)\left(y_{2}+2 y_{4}\right)} \\
& \cdot \tilde{\psi}_{\gamma_{1}, \beta_{1}}\left(-y_{2}-y_{4}\right) \tilde{\psi}_{\gamma_{3}, \beta_{3}}\left(y_{2}+2 y_{4}\right) \prod_{i \in\{2,4\}} \psi_{\gamma_{i}, \beta_{i}}\left(y_{i}\right) \\
\simeq & \int_{\mathbb{R}^{2}} \mathrm{~d} y_{2} \mathrm{~d} y_{4} e^{\pi \mathrm{i} y_{4}^{2}} \psi_{\beta_{1}, \alpha_{1}}\left(-y_{2}-y_{4}\right) \psi_{\beta_{3}, \alpha_{3}}\left(y_{2}+2 y_{4}\right) \prod_{i \in\{2,4\}} \psi_{\gamma_{i}, \beta_{i}}\left(y_{i}\right)
\end{aligned}
$$

where we integrated over $y_{1}$ and $y_{3}$ by using the Fourier transformation

$$
\tilde{\psi}_{\alpha, \beta}(x)=\int_{\mathbb{R}} \mathrm{d} y \psi_{\alpha, \beta}(x) e^{-2 \pi \mathbf{i} x y}
$$


and used the equality

$$
\tilde{\psi}_{\alpha, \beta}(x) \simeq \psi_{\beta, \pi-\alpha-\beta}(x) e^{\pi \mathrm{i} x^{2}}
$$

We remark that the "charged" pentagon identity (20) of [AK14] can be equivalently written in the form ${ }^{1}$

$$
\begin{aligned}
& \bar{\psi}_{c_{4}, a_{4}}(y) \psi_{b_{2}, a_{2}}(u) \bar{\psi}_{c_{0}, a_{0}}(x) \delta(x-u+y) \\
\simeq & \int_{\mathbb{R}^{2}} Q_{u, v}^{x, y, z} \bar{\psi}_{b_{1}, c_{1}}(v) \psi_{a_{3}, c_{3}}(z) \mathrm{d} v \mathrm{~d} z
\end{aligned}
$$

and also in the form

$$
\bar{\psi}_{c_{4}, a_{4}}(y) \psi_{b_{2}, a_{2}}(x+y) \bar{\psi}_{c_{0}, a_{0}}(x) \simeq \int_{\mathbb{R}} e^{2 \pi \mathrm{i} x z} \bar{\psi}_{b_{1}, c_{1}}(y+z) \psi_{a_{3}, c_{3}}(z) \mathrm{d} z
$$

where

$$
\begin{aligned}
& \left(a_{i}, b_{i}, c_{i}\right) \in \mathbb{R}_{>0}^{3}, \quad a_{i}+b_{i}+c_{i}=\pi, \quad i \in[4], \\
& a_{1}=a_{0}+a_{2}, \quad a_{3}=a_{2}+a_{4}, \\
& c_{1}=c_{0}+a_{4}, \quad c_{3}=a_{0}+c_{4}, \quad c_{2}=c_{1}+c_{3} .
\end{aligned}
$$

By using the symmetry relation

$$
\bar{\psi}_{\alpha, \beta}(x) \simeq \psi_{\beta, \alpha}(-x) e^{\pi \mathbf{i} x^{2}}
$$

one can apply (B.25) and reduce the eventual multiplicity of the integral in (B.22) from two to one, provided the condition

$$
w\left(e_{2}\right)-w\left(e_{0}\right)=\gamma_{1}+\alpha_{2}+\alpha_{4}=2 \pi
$$

is satisfied. That condition is satisfied due to (B.14). At the present stage, we continue our calculation imposing only the condition (B.29) and the

\footnotetext{
${ }^{1}$ As the charges of [AK14] are the dihedral angles measured by arc lengths of a circle of circumference 1 , the function $\psi_{a, c}(x)$ of [AK14], up to a phase independent of $x$, is $\psi_{\frac{a}{2 \pi}}, \frac{c}{2 \pi}(x)$ in the notation we use here.
} 
inequalities

$$
\alpha_{2}>\alpha_{1}+\gamma_{4}, \quad \alpha_{4}>\alpha_{1}+\gamma_{2}
$$

Denoting

$$
\begin{aligned}
& \alpha_{5}=\alpha_{2}-\alpha_{1}-\gamma_{4}, \quad \beta_{5}=\beta_{2}+\gamma_{4} \\
& \alpha_{6}=\gamma_{2}+\beta_{4}, \quad \beta_{6}=\alpha_{1}+\gamma_{4} \\
& \gamma_{i}=\pi-\alpha_{i}-\beta_{i}, \quad i \in\{5,6\}
\end{aligned}
$$

and going back to the last part of Equation (B.22), we further write

$$
\begin{aligned}
\tilde{Z}(X) & \simeq \int_{\mathbb{R}^{2}} \mathrm{~d} y_{2} \mathrm{~d} y_{4} e^{-\pi \mathrm{i} y_{2}^{2}} \psi_{\beta_{1}, \alpha_{1}}\left(-y_{2}-y_{4}\right) \psi_{\beta_{3}, \alpha_{3}}\left(y_{2}+2 y_{4}\right) \prod_{i \in\{2,4\}} \bar{\psi}_{\beta_{i}, \gamma_{i}}\left(-y_{i}\right) \\
& \simeq \int_{\mathbb{R}^{3}} \mathrm{~d} y_{2} \mathrm{~d} y_{4} \mathrm{~d} z e^{-\pi \mathrm{i}\left(y_{2}^{2}+2 y_{2} z\right)} \psi_{\beta_{3}, \alpha_{3}}\left(y_{2}+2 y_{4}\right) \bar{\psi}_{\alpha_{5}, \beta_{5}}\left(z-y_{4}\right) \psi_{\beta_{6}, \alpha_{6}}(z) \\
& \simeq \int_{\mathbb{R}^{3}} \mathrm{~d} y_{2} \mathrm{~d} y_{4} \mathrm{~d} z e^{-\pi \mathrm{i}\left(y_{2}+z\right)^{2}} \psi_{\beta_{3}, \alpha_{3}}\left(y_{2}+2 y_{4}\right) \bar{\psi}_{\alpha_{5}, \beta_{5}}\left(z-y_{4}\right) \bar{\psi}_{\alpha_{6}, \beta_{6}}(-z)
\end{aligned}
$$

where we used twice the symmetry relation (B.28) in the first equality, the charged pentagon relation (B.25) with $\left(c_{0}, a_{0}\right)=\left(\beta_{2}, \gamma_{2}\right),\left(b_{2}, a_{2}\right)=\left(\beta_{1}, \alpha_{1}\right)$, $\left(c_{4}, a_{4}\right)=\left(\beta_{4}, \gamma_{4}\right),\left(b_{1}, c_{1}\right)=\left(\alpha_{5}, \beta_{5}\right)$ and $\left(a_{3}, c_{3}\right)=\left(\beta_{6}, \alpha_{6}\right)$ in the second one, and again the symmetry relation (B.28) in the last one.

We continue by shifting the integration variables $y_{2} \mapsto y_{2}-2 y_{4}$ followed by $y_{4} \mapsto y_{4}+z$ in the above equation. It follows that

$$
\begin{aligned}
\tilde{Z}(X) & \simeq \int_{\mathbb{R}^{3}} \mathrm{~d} y_{2} \mathrm{~d} y_{4} \mathrm{~d} z e^{-\pi \mathrm{i}\left(y_{2}-z-2 y_{4}\right)^{2}} \psi_{\beta_{3}, \alpha_{3}}\left(y_{2}\right) \bar{\psi}_{\alpha_{5}, \beta_{5}}\left(-y_{4}\right) \bar{\psi}_{\alpha_{6}, \beta_{6}}(-z) \\
& \simeq \int_{\mathbb{R}^{3}} \mathrm{~d} y_{2} \mathrm{~d} y_{4} \mathrm{~d} z e^{\pi \mathrm{i}\left(2 z-y_{2}+2 y_{4}\right)\left(y_{2}-2 y_{4}\right)} \psi_{\beta_{3}, \alpha_{3}}\left(y_{2}\right) \bar{\psi}_{\alpha_{5}, \beta_{5}}\left(-y_{4}\right) \psi_{\beta_{6}, \alpha_{6}}(z) \\
& =\int_{\mathbb{R}^{2}} \mathrm{~d} y_{2} \mathrm{~d} y_{4} e^{-\pi \mathrm{i}\left(2 y_{4}-y_{2}\right)^{2}} \psi_{\beta_{3}, \alpha_{3}}\left(y_{2}\right) \bar{\psi}_{\alpha_{5}, \beta_{5}}\left(-y_{4}\right) \tilde{\psi}_{\beta_{6}, \alpha_{6}}\left(2 y_{4}-y_{2}\right) \\
& \simeq \int_{\mathbb{R}^{2}} \mathrm{~d} y_{2} \mathrm{~d} y_{4} \psi_{\beta_{3}, \alpha_{3}}\left(y_{2}\right) \bar{\psi}_{\alpha_{5}, \beta_{5}}\left(-y_{4}\right) \psi_{\alpha_{6}, \gamma_{6}}\left(2 y_{4}-y_{2}\right)
\end{aligned}
$$

where we used the symmetry relation (B.28) backwards in the second equality, the definition of the Fourier transformation (B.23) in the third one, and Formula (B.24) in the last one. 
Now, by using the Fourier transformation (B.23) and its inverse, as well as the Formulae (B.24) and (B.28), we arrive at a single integral expression:

$$
\begin{aligned}
\tilde{Z}(X) & \simeq \int_{\mathbb{R}^{3}} \mathrm{~d} y_{2} \mathrm{~d} y_{4} \mathrm{~d} t e^{2 \pi \mathrm{i}\left(2 y_{4}-y_{2}\right) t} \psi_{\beta_{3}, \alpha_{3}}\left(y_{2}\right) \bar{\psi}_{\alpha_{5}, \beta_{5}}\left(-y_{4}\right) \tilde{\psi}_{\alpha_{6}, \gamma_{6}}(t) \\
& =\int_{\mathbb{R}} \mathrm{d} t \tilde{\psi}_{\beta_{3}, \alpha_{3}}(t) \overline{\tilde{\psi}}_{\alpha_{5}, \beta_{5}}(-2 t) \tilde{\psi}_{\alpha_{6}, \gamma_{6}}(t) \\
& \simeq \int_{\mathbb{R}} \mathrm{d} t e^{-2 \pi \mathrm{i} t^{2}} \psi_{\alpha_{3}, \gamma_{3}}(t) \bar{\psi}_{\beta_{5}, \gamma_{5}}(-2 t) \psi_{\gamma_{6}, \beta_{6}}(t) \\
& \simeq \int_{\mathbb{R}} \mathrm{d} t e^{2 \pi \mathrm{i} t^{2}} \psi_{\alpha_{3}, \gamma_{3}}(t) \psi_{\gamma_{5}, \beta_{5}}(2 t) \psi_{\gamma_{6}, \beta_{6}}(t)
\end{aligned}
$$

Introducing a new complex integration variable $z=t-c_{\mathrm{b}}+c_{\mathrm{b}} \frac{\beta_{3}}{\pi}$ and using Formula (B.13), we rewrite our integral in an explicit form by using the total dihedral angles:

$$
\begin{aligned}
& \tilde{Z}(X) \simeq \int_{\mathbb{R}-c_{\mathrm{b}}+c_{\mathrm{b}} \frac{\beta_{3}}{\pi}} \mathrm{d} z \frac{e^{2 \mathrm{i}\left(z+c_{\mathrm{b}}-c_{\mathrm{b}} \frac{\beta_{3}}{\pi}\right)\left(\pi z+c_{\mathrm{b}}\left(\gamma_{3}-2 \gamma_{5}-\gamma_{6}\right)\right)}}{\Phi_{\mathrm{b}}(z) \Phi_{\mathrm{b}}\left(c_{\mathrm{b}}+2 z+c_{\mathrm{b}} \frac{\alpha_{5}-2 \beta_{3}}{\pi}\right) \Phi_{\mathrm{b}}\left(z+c_{\mathrm{b}} \frac{\alpha_{6}-\beta_{3}}{\pi}\right)} \\
& \simeq \int_{\mathbb{R}-c_{\mathrm{b}}+c_{\mathrm{b}} \frac{\beta_{3}}{\pi}} \mathrm{d} z \frac{e^{2 \pi \mathrm{i} z^{2}} e^{2 \mathrm{i} c_{\mathrm{b}} z\left(\pi-\beta_{3}+\gamma_{3}-2 \gamma_{5}-\gamma_{6}\right)}}{\Phi_{\mathrm{b}}(z) \Phi_{\mathrm{b}}\left(c_{\mathrm{b}}+2 z+c_{\mathrm{b}} \frac{\alpha_{5}-2 \beta_{3}}{\pi}\right) \Phi_{\mathrm{b}}\left(z+c_{\mathrm{b}} \frac{\alpha_{6}-\beta_{3}}{\pi}\right)} \\
& =\int_{\mathbb{R}-c_{\mathrm{b}}+c_{\mathrm{b}} \frac{\beta_{3}}{\pi}} \mathrm{d} z \frac{e^{2 \pi \mathrm{i} z^{2}} e^{2 \mathrm{i} c_{\mathrm{b}} z\left(\pi-\alpha_{1}-\gamma_{2}-\beta_{3}+\gamma_{3}-\alpha_{4}\right)}}{\Phi_{\mathrm{b}}(z) \Phi_{\mathrm{b}}\left(c_{\mathrm{b}}+2 z+c_{\mathrm{b}} \frac{\alpha_{2}-\alpha_{1}-\gamma_{4}-2 \beta_{3}}{\pi}\right)} \\
& \frac{1}{\Phi_{\mathrm{b}}\left(z+c_{\mathrm{b}} \frac{\gamma_{2}+\beta_{4}-\beta_{3}}{\pi}\right)} \\
& =\int_{\mathbb{R}-c_{\mathrm{b}}+c_{\mathrm{b}} \frac{\beta_{3}}{\pi}} \mathrm{d} z \frac{e^{2 \pi \mathrm{i} z^{2}} e^{2 \mathrm{i} c_{\mathrm{b}} z\left(w\left(e_{0}\right)+w\left(e_{1}\right)+w\left(e_{5}\right)-4 \pi\right)}}{\Phi_{\mathrm{b}}(z) \Phi_{\mathrm{b}}\left(c_{\mathrm{b}}+2 z+c_{\mathrm{b}} \frac{w\left(e_{5}\right)-w\left(e_{3}\right)-w\left(e_{0}\right)}{\pi}\right)} \\
& \cdot \frac{1}{\Phi_{\mathrm{b}}\left(z+c_{\mathrm{b}} \frac{2 \pi-w\left(e_{0}\right)-w\left(e_{3}\right)}{\pi}\right)} \text {. }
\end{aligned}
$$

We observe that conditions (B.14) imply that the three linear combinations of total dihedral angles entering the integrand vanish. Formula (B.15) is now obtained by using the analyticity of the integrand to reduce the complex shift of the integration line to arbitrarily small value of the same sign and applying the inversion relation (A.2) followed by negation of the integration variable. 


\section{References}

[AKMV05] Mina Aganagic, Albrecht Klemm, Marcos Mariño and Cumrun Vafa, The topological vertex. Comm. Math. Phys., 254 (2005), no. $2,425-478$.

[AK] Jørgen E. Andersen and R. M. Kashaev, Complex Quantum Chern-Simons. arXiv:1409.1208, preprint, 2014.

[AK14] Jørgen E. Andersen and R. M. Kashaev, A TQFT from Quantum Teichmüller theory. Comm. Math. Phys., 330 (2014), no. 3, 887-934.

[BB07] Stephane Baseilhac and Riccardo Benedetti, Quantum hyperbolic geometry. Algebr. Geom. Topol., 7 (2007), 845-917.

[Cul] Marc Culler, A table of A-polynomials. http://www.math.uic. edu/Apolynomials.

[CDW] Marc Culler, Nathan M. Dunfield and Jeffery R. Weeks, SnapPy. http://www . math. uic.edu/t3m/SnapPy.

[Dim14a] Tudor Dimofte, Complex Chern-Simons theory at level $k$ via the 3d-3d correspondence. arXiv:1409.0857, preprint, 2014.

[Dim14b] Tudor Dimofte, Quantum Modularity and Complex ChernSimons theory. Preprint, 2014.

[DG13] Tudor Dimofte and Stavros Garoufalidis, The quantum content of the gluing equations. Geom. Topol., 17 (2013), no. 3, 12531315 .

[DGLZ09] Tudor Dimofte, Sergei Gukov, Jonatan Lenells and Don Zagier, Exact results for perturbative Chern-Simons theory with complex gauge group. Commun. Number Theory Phys., 3 (2009), no. 2, 363-443.

[Fad95] L. D. Faddeev, Discrete Heisenberg-Weyl group and modular group. Lett. Math. Phys., 34 (1995), no. 3, 249-254.

[FK94] L. D. Faddeev and R. M. Kashaev, Quantum dilogarithm. Modern Phys. Lett. A, 9 (1994), no. 5, 427-434.

[GGZ14] Stavros Garoufalidis, Matthias Rolf Dietrich Goerner, Sr. and Christian K. Zickert, The Ptolemy field of 3-manifoldrepresentations. arXiv:1401.5542, preprint, 2014. 
[GK] Stavros Garoufalidis and R. M. Kashaev, From state-integrals to q-series. Math. Research Letters, in press.

[GZa] Stavros Garoufalidis and Don Zagier, Asymptotics of quantum knot invariants. Preprint, 2013.

[GZb] Stavros Garoufalidis and Don Zagier, Empirical relations between q-series and kashaev's invariant of knots. Preprint, 2013.

[GZ07] Sebastian Goette and Christian K. Zickert, The extended Bloch group and the Cheeger-Chern-Simons class. Geom. Topol., 11 (2007), 1623-1635.

[Hik01] Kazuhiro Hikami, Hyperbolic structure arising from a knot invariant. Internat. J. Modern Phys. A, 16 (2001), no. 19, 33093333.

[Kas94] R. M. Kashaev, Quantum dilogarithm as a 6j-symbol. Modern Phys. Lett. A, 9 (1994), no. 40, 3757-3768.

[Kas95] R. M. Kashaev, A link invariant from quantum dilogarithm. Modern Phys. Lett. A, 10 (1995), no. 19, 1409-1418.

[Kas97] R. M. Kashaev, The hyperbolic volume of knots from the quantum dilogarithm. Lett. Math. Phys., 39 (1997), no. 3, 269-275.

[Kas99] R. M. Kashaev, Quantum hyperbolic invariants of knots. Discrete integrable geometry and physics (Vienna, 1996), Oxford Lecture Ser. Math. Appl., Vol. 16, Oxford Univ. Press, New York, 1999, pp. 343-359.

[KLV12] R. M. Kashaev, Feng Luo and Grigory Vartanov, A TQFT of Turaev-Viro type on shaped triangulations. arXiv:1210.8393, preprint, 2012.

[KMS93] R. M. Kashaev, V. V. Mangazeev and Yu. G. Stroganov, Starsquare and tetrahedron equations in the Baxter-Bazhanov model. Internat. J. Modern Phys. A, 8 (1993), no. 8, 1399-1409.

[Neu04] Walter D. Neumann, Extended Bloch group and the CheegerChern-Simons class. Geom. Topol., 8 (2004), 413-474 (electronic).

[ORV06] Andrei Okounkov, Nikolai Reshetikhin and Cumrun Vafa, Quantum Calabi-Yau and classical crystals. The unity of mathematics, Progr. Math., Vol. 244, Birkhäuser Boston, Boston, MA, 2006, pp. 597-618. 
[Zag10] Don Zagier, Quantum modular forms. Quanta of maths, Clay Math. Proc., Vol. 11, Amer. Math. Soc., Providence, RI, 2010, pp. 659-675.

School of Mathematics, Georgia Institute of Technology

AtlantA, GA 30332-0160, USA

E-mail address: stavros@math.gatech.edu

Section de Mathématiques, Université de Genève

2-4 Rue du Lièvre, Case Postale 64, 1211 Genève 4, Switzerland

E-mail address: Rinat.Kashaev@unige.ch

Received December 30, 2014 